\title{
Orbits and phase transitions in the multifractal spectrum
}

\author{
Thomas Nowotny, Heiko Patzlaff and Ulrich Behn \\ Institut für Theoretische Physik, Universität Leipzig \\ Augustusplatz 10, 04109 Leipzig, Germany
}

\begin{abstract}
We consider the one dimensional classical Ising model in a symmetric dichotomous random field. The problem is reduced to a random iterated function system for an effective field. The $D_{q}$-spectrum of the invariant measure of this effective field exhibits a sharp drop of all $D_{q}$ with $q<0$ at some critical strength of the random field. We introduce the concept of orbits which naturally group the points of the support of the invariant measure. We then show that the pointwise dimension at all points of an orbit has the same value and calculate it for a class of periodic orbits and their so-called offshoots as well as for generic orbits in the non-overlapping case. The sharp drop in the $D_{q}$-spectrum is analytically explained by a drastic change of the scaling properties of the measure near the points of a certain periodic orbit at a critical strength of the random field which is explicitly given. A similar drastic change near the points of a special family of periodic orbits explains a second, hitherto unnoticed transition in the $D_{q^{-}}$ spectrum. As it turns out, a decisive role in this mechanism is played by a specific offshoot. We furthermore give rigorous upper and/or lower bounds on all $D_{q}$ in a wide parameter range. In most cases the numerically obtained $D_{q}$ coincide with either the upper or the lower bound. The results in this paper are relevant for the understanding of random iterated function systems in the case of moderate overlap in which periodic orbits with weak singularity can play a decisive role.
\end{abstract}

PACS numbers: 05.45.Df, 05.50.+q, 75.10.Nr, 05.70.Fh

\section{Introduction}

The properties of multifractal measures have attracted a lot of interest over the past two decades. Multifractals naturally appear in a variety of physical and mathematical contexts. From the beginning the one dimensional random field [1]- 17] and random exchange [18-20] Ising models were prominent examples. In treating these systems some reduction scheme for the partition function like the transfer matrix method [1, 2, 17, 18] or a method introduced by Ruján [9] usually is used [1]-15] to obtain a random iterated function system (RIFS) for a local effective field. This leads via a Frobenius-Perron or Chapman-Kolmogorov equation to an invariant measure which typically is a multifractal. Similar structures arise in other one dimensional disordered systems like phonons 21] or electrons [22, 23] in random potentials, cf. also [24].

In the early investigations of the random field Ising model the language of multifractals had not yet been developed and the results focused apart from calculating the free energy on the structure of the support of the invariant measure [1]- [8] and the ground state properties of the system $[5,6,8,12,18]$. More recently the uniqueness of 
Gibbs measures and exact ground state properties were investigated [16]. A connection to domain theory was established in 25$]$.

The invariant measure of the local effective field in the random field Ising model is a multifractal [8]. In various works the generalized box dimensions (generalized Rényi dimensions) $D_{q}$ [26] of this measure were calculated for special $q$ [4, 10], with perturbation expansions [7, 10, 11, 27] or numerical approximations [10, 13, 27]. Other authors focused on different concepts like the order- $q$ free energy and its fluctuations [19, 20] or correlation functions [17].

The systematic numerical investigation of the dependence of $D_{q}$ on the strength of the local random fields 13 revealed the surprising feature of discontinuities (phase transitions) in the $D_{q}$ with negative $q$.

Almost all features of the $D_{q}$-spectrum have by now been understood analytically. The most drastic transition in the $D_{q}$-spectrum, the sharp drop of all generalized dimensions $D_{q}$ with $q<0$ at some critical field strength $h_{c}^{(2)}$, also present in the context of a special model of neural networks [28, 29], was explained on a phenomenological level by the disappearance of deep cuts in the measure density at $h \leq h_{c}^{(2)}$ [14, 29]. This disappearance of deep cuts in the measure density can be explained analytically by close investigation of the obtained nonlinear RIFS.

In this paper we complete the analysis of the transition begun in [15, 30 and explicitly calculate the critical field strength $h_{c}^{(2)}$ of the transition. The result is obtained by generalizing the analysis of the singularity (pointwise dimension) at fixed points to the singularity of orbits. Further application to a special family of periodic orbits explains a so far unnoticed smaller drop in the $D_{q}$-spectrum at a critical field strength $h_{c}^{(2 a)}$ which became observable because of increased precision in the numerical generation of the $D_{q}$-spectrum. Furthermore, the concept of orbits and their singularity also allows to give bounds on $D_{q}$ for any $q$ and the exact value of $D_{ \pm \infty}$ in a wide parameter region of the random field strength $h$. The computation of $D_{ \pm \infty}$ generalizes earlier results in [10].

Similar approaches and arguments may be found in the mathematical literature. In 31] parabolic function systems with overlaps are considered, [32] concentrates on measures obtained by infinite Bernoulli convolutions and [33] investigates generalized dimensions $D_{q}$ of measures on general self-affine sets.

In the following we consider the one-dimensional random field Ising model [1]- 17 ] with the Hamiltonian

$$
H_{N}=-J \sum_{i=1}^{N-1} s_{i} s_{i+1}-\sum_{i=1}^{N} h_{i} s_{i},
$$

in which $s_{i}$ denotes the classical spin at site $i$ which takes values 1 or -1 and $J$ is the exchange energy of adjacent spins. The local magnetic fields $\left\{h_{i}\right\}$ at the sites $i=1, \ldots, N$ are independent identically distributed random variables. We restrict ourselves to dichotomous symmetric distributions, i.e. to probability densities with Dirac masses at $\pm h$,

$$
\rho\left(h_{i}\right)=\frac{1}{2} \delta\left(h_{i}-h\right)+\frac{1}{2} \delta\left(h_{i}+h\right), \quad h \in \mathbb{R}^{+} .
$$

An iterative reformulation of the canonical partition function yields the partition function of a single spin in an effective external random field $x_{1}^{(N)}$ (the effective field 
at site 1 in a chain of $N$ spins) which is given by an iterative map [4,

$$
\begin{aligned}
& x_{i}^{(N)}=h_{i}+A\left(x_{i+1}^{(N)}\right), \quad x_{N+1}^{(N)}=0 \\
& A(x)=\frac{1}{2 \beta} \ln \left(\frac{\cosh \beta(x+J)}{\cosh \beta(x-J)}\right),
\end{aligned}
$$

where $\beta$ denotes the inverse temperature f The iteration is illustrated in figure 1. As a shorthand we introduce

$$
f_{\sigma}(x):=\sigma h+A(x), \quad \sigma \in\{+,-\}
$$

such that the recursion (3) reads $x_{i}^{(N)}=f_{\sigma_{i}}\left(x_{i+1}^{(N)}\right)$ with $h_{i}=: \sigma_{i} h$. By the reformulation of the canonical partition function we are thus led to a RIFS with smooth, strictly monotonously growing, contractive functions $\left\{f_{+}, f_{-}\right\}=\{A+h, A-h\}$ and probabilities $\left\{p_{+}, p_{-}\right\}=\left\{\frac{1}{2}, \frac{1}{2}\right\}$. When viewing (3) as a RIFS we will also write $x_{n}$ instead of $x_{i}^{(N)}$ for the value of the effective field after $n=N-i+1$ iterations. Please note that the transition from $N$ to $N+1$ spins implies prepending functions to the composition of functions, i.e. we need to consider $x_{n}=f_{\sigma_{1}} \circ \ldots \circ f_{\sigma_{n}}(x)$, $x_{n+1}=f_{\sigma_{1}} \circ \ldots \circ f_{\sigma_{n}} \circ f_{\sigma_{n+1}}(x)$, etc.

We introduce a symbolic dynamic in the obvious way: Let $\Sigma_{n}$ be the set of finite sequences $\{\sigma\}_{n}$ of $n$ symbols $\sigma_{i} \in\{+,-\}, i=1, \ldots, n$ and $\Sigma_{\infty}$ the set of all infinite sequences $\{\sigma\}$. Given $\{\sigma\}$ we will write $\{\sigma\}_{n}$ for the head of the $n$ leftmost symbols in $\{\sigma\}$. By $f_{\{\sigma\}_{n}}$ we denote the composition of the $n$ functions $f_{\sigma_{i}}, i=1, \ldots, n$, i.e. $f_{\{\sigma\}_{n}}=f_{\sigma_{1}} \circ f_{\sigma_{2}} \circ \ldots \circ f_{\sigma_{n}}$. The above mentioned properties of $f_{+}$and $f_{-}$imply the following facts:

- The fixed points $x_{+}^{*}$ and $x_{-}^{*}$ with $f_{+}\left(x_{+}^{*}\right)=x_{+}^{*}$ and $f_{-}\left(x_{-}^{*}\right)=x_{-}^{*}$ exist.

- The interval $I=\left[x_{-}^{*}, x_{+}^{*}\right]$ is the smallest interval with $f_{\sigma}(I) \subseteq I$.

- The $\operatorname{limit}_{\lim _{n \rightarrow \infty}} f_{\{\sigma\}_{n}}\left(x_{0}\right)$ exists for any $\{\sigma\} \in \Sigma_{\infty}$ and $x_{0} \in I$ and does not depend on $x_{0}$. We thus can define $x_{\{\sigma\}}^{*}:=\lim _{n \rightarrow \infty} f_{\{\sigma\}_{n}}\left(x_{0}\right)$ and the (constant) function $f_{\{\sigma\}}: I \rightarrow I, f_{\{\sigma\}}(x):=x_{\{\sigma\}}^{*}$. Through this definition $x_{\{\sigma\}}^{*}$ is the unique fixed point of $f_{\{\sigma\}}$.

- We denote the fixed points of finite compositions $f_{\{\sigma\}_{n}}$ by $x_{\{\sigma\}_{n}}^{*}$. If $\{\sigma\}$ is periodic with period $n$ then $x_{\{\sigma\}}^{*}=x_{\{\sigma\}_{n}}^{*}$.

- We name the $n$-fold images $I_{\{\sigma\}_{n}}:=f_{\{\sigma\}_{n}}(I)$ of the invariant interval $I$ bands (of order $n$ ). For given $\{\sigma\}$ they have the inclusion property $I_{\{\sigma\}_{n}} \subset I_{\{\sigma\}_{m}}$ for $n>m$. Furthermore, the construction implies $x_{\{\sigma\}}^{*} \in I_{\{\sigma\}_{n}}$ for all $n$.

- If the first order bands $I_{+}$and $I_{-}$do not overlap, $I_{+} \cap I_{-}=\emptyset$, none of the higher order bands overlap, $I_{\{\sigma\}_{n}} \cap I_{\{\tilde{\sigma}\}_{n}}=\emptyset$. We call this the non-overlapping case and denote the first order gap by $\Delta:=\left[f_{-}\left(x_{+}^{*}\right), f_{+}\left(x_{-}^{*}\right)\right]$. The inverse statement is also true. If the first order bands overlap the corresponding higher order bands also overlap. This situation is illustrated in figure 11. We denote the first order overlap by $O:=I_{+} \cap I_{-}$.

The RIFS (2, 3) induces a probability density $p_{n}$ for the effective field $x_{n}$. We write $P_{n}(x):=\int_{0}^{x} p_{n}(\xi) d \xi$ for the corresponding distribution function and $\mu_{n}$ for

\pm Note, that we find it convenient to use a slightly different notation than in previous work as e.g. [6, 8, 15. 
a)

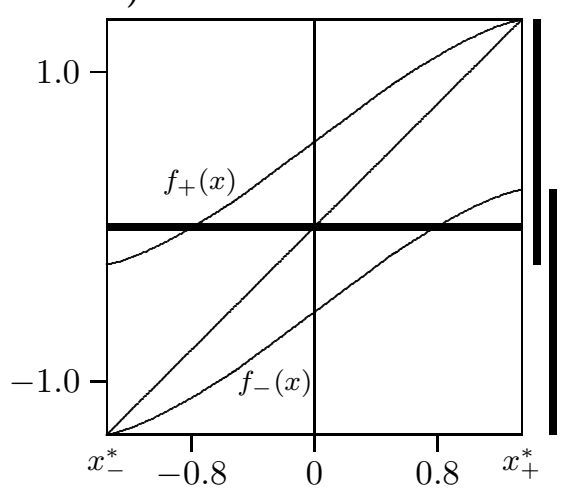

b)

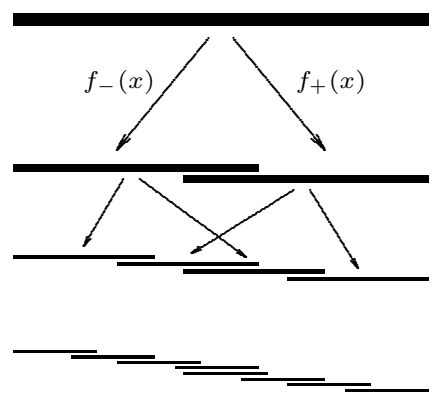

Figure 1. (a) Mapping in the case of overlapping bands $I_{+}=f_{+}(I)$ and $I_{-}=f_{-}(I)$. In (b) the first few images of the interval $I$ are shown. The increasing complexity of the band structure is obvious $(h=0.55, \beta=1, J=1)$.

the corresponding measure. $P_{n}, p_{n}$ and $\mu_{n}$ can iteratively be constructed using the Frobenius-Perron (Chapman-Kolmogorov) equation induced by (3) which reads for $P_{n}$

$$
P_{n}(x)=\int d h \rho(h) P_{n-1}\left(A^{-1}(x-h)\right)=\sum_{\sigma= \pm} \frac{1}{2} P_{n-1}\left(f_{\sigma}^{-1}(x)\right)
$$

with $P_{0}(x)=\Theta(x), \Theta$ being the Heaviside function. This choice of $P_{0}$ encodes the free boundary conditions chosen in (3). Again the properties of $f_{+}$and $f_{-}$imply some direct consequences:

- The Frobenius-Perron equation has a unique fixed point $P_{\infty}\left(\mu_{\infty}\right)$ [5, 34] and the invariant measure $\mu_{\infty}$ is ergodic.

- The reiterated application of the Frobenius-Perron equation to an arbitrary initial measure $\mu_{0}$ (distribution $P_{\infty}$ ) converges to $\mu_{\infty}\left(P_{0}\right)$ in Hutchinson topology or, as $I$ is compact, equivalently in the weak topology of measures [34]. Thus, $\mu_{\infty}$ $\left(P_{\infty}\right)$ is the measure (distribution) of the effective field $x$ in the thermodynamic limit $N \rightarrow \infty$ for any boundary condition.

- The explicit form of the $n$-th iterate $P_{n}(x)$ reads

$$
P_{n}(x)=\sum_{\{\sigma\}_{n}} \frac{1}{2^{n}} P_{0}\left(f_{\{\sigma\}_{n}}^{-1}(x)\right),
$$

which is in the limit $n \rightarrow \infty$ a path integral in the space of symbolic dynamics. In the non-overlapping case the sum on the right hand side of (6) has only one term for each $x$. In the overlapping case typically more than one term contributes for each $x$. There are however $x \in \operatorname{supp} \mu_{\infty}$ for which still only one term contributes.

- The support supp $\mu_{\infty} \subseteq I$ is the attractor of the RIFS $\left\{f_{+}, f_{-}\right\}$[34].

- For any $x \in \operatorname{supp} \mu_{\infty}$ one can find a sequence $\{\sigma\}$ with $x=x_{\{\sigma\}}^{*}$. In the nonoverlapping case this relation between $\Sigma_{\infty}$ and supp $\mu_{\infty}$ is one to one. 
In the following we closely investigate the multifractal properties of the invariant measure $\mu_{\infty}$. To this end we study the generalized box counting dimensions

$$
D_{q}=\frac{1}{q-1} \lim _{\varepsilon \rightarrow 0} \frac{\ln \left(\sum_{i} \mu_{i}^{q}\right)}{\ln \varepsilon},
$$

where $\mu_{i}=\mu_{\infty}\left(B_{\varepsilon}\left(x_{i}\right)\right)$ are the measures of boxes (intervals) of size $\varepsilon$ covering supp $\mu_{\infty}$, and the pointwise dimension

$$
D_{p}(x)=\lim _{\varepsilon \rightarrow 0} \frac{\ln \mu_{\infty}\left(B_{\varepsilon}(x)\right)}{\ln \varepsilon}
$$

at individual points $x \in \operatorname{supp} \mu_{\infty}$. We will synonymously use the singularity $\alpha:=$ $D_{p}-1$. The intricate interplay between these quantities will explain the transitions in the $D_{q}$-spectrum mentioned above. The obtained characterization of $\mu_{\infty}$, the measure of the effective field $x$, is a necessary prerequisite for the more complicated treatment of the distribution of physical quantities like the local magnetisation which will follow in a later publication.

The paper is organised as follows. In subsection 1.1 we briefly review the known results about prominent features of the $D_{q}$-spectrum. In section 2 the concept of orbits and their singularity is introduced and in 2.1 this singularity is calculated for a class of periodic orbits and their so-called offshoots. In subsection 2.2 we calculate the generic singularity of arbitrary orbits in the non-overlapping case. In subsection 2.3 we treat the overlapping case and discuss effects occurring if points of an orbit enter the overlap. The results are used to explain the transition in the $D_{q}$-spectrum at $h_{c}^{(2)}$ and calculate $h_{c}^{(2)}$ as a function of temperature $T$ and coupling strength $J$ explicitly. We then apply a similar analysis to the transition at $h_{c}^{(2 a)}$ in subsection 2.4. In section 3 we give the extended lower and upper bounds on the $D_{q}$-spectrum obtained from the analysis of the singularity of specific orbits. Finally, some conclusions are drawn in section 1.

\subsection{Known results}

In this subsection we summarize in short previous work on phase transitions in the invariant measure $\mu_{\infty}$. For large $h$ the support of $\mu_{\infty}$ is non-connected and similar to a multi-scale Cantor set [8].

At a critical value $h_{c}^{(1)}$ of $h$ the support of $\mu_{\infty}$ becomes connected for all $h \leq h_{c}^{(1)}$ [2]. The value of $h_{c}^{(1)}$ is determined by the overlap condition for the first bands, $f_{-}\left(x_{+}^{*}\right)=f_{+}\left(x_{-}^{*}\right)$. This results in $[13$

$$
h_{c}^{(1)}=\frac{1}{2 \beta} \operatorname{arcosh}\left(\left(e^{2 \beta J}-1\right) / 2\right) .
$$

The transition can be seen in the densities $p_{n}$ of the approximations $\mu_{n}$ of the invariant measure $\mu_{\infty}$ [13]. In the $D_{q}$-spectrum the transition is visible as the point where $D_{0}$ becomes 1 , cf. figure 2 .

At $h_{c}^{(3)} \leq h_{c}^{(1)}$ the invariant measure density jumps from infinity to zero at the boundary $x_{+}^{*}$ and $x_{-}^{*}$ of $I$ [13]. This is an effect solely depending on the scaling of the measure at the fixed points $x_{-}^{*}$ and $x_{+}^{*}$. The measure density $p_{n}\left(x_{ \pm}^{*}\right)$ diverges for 


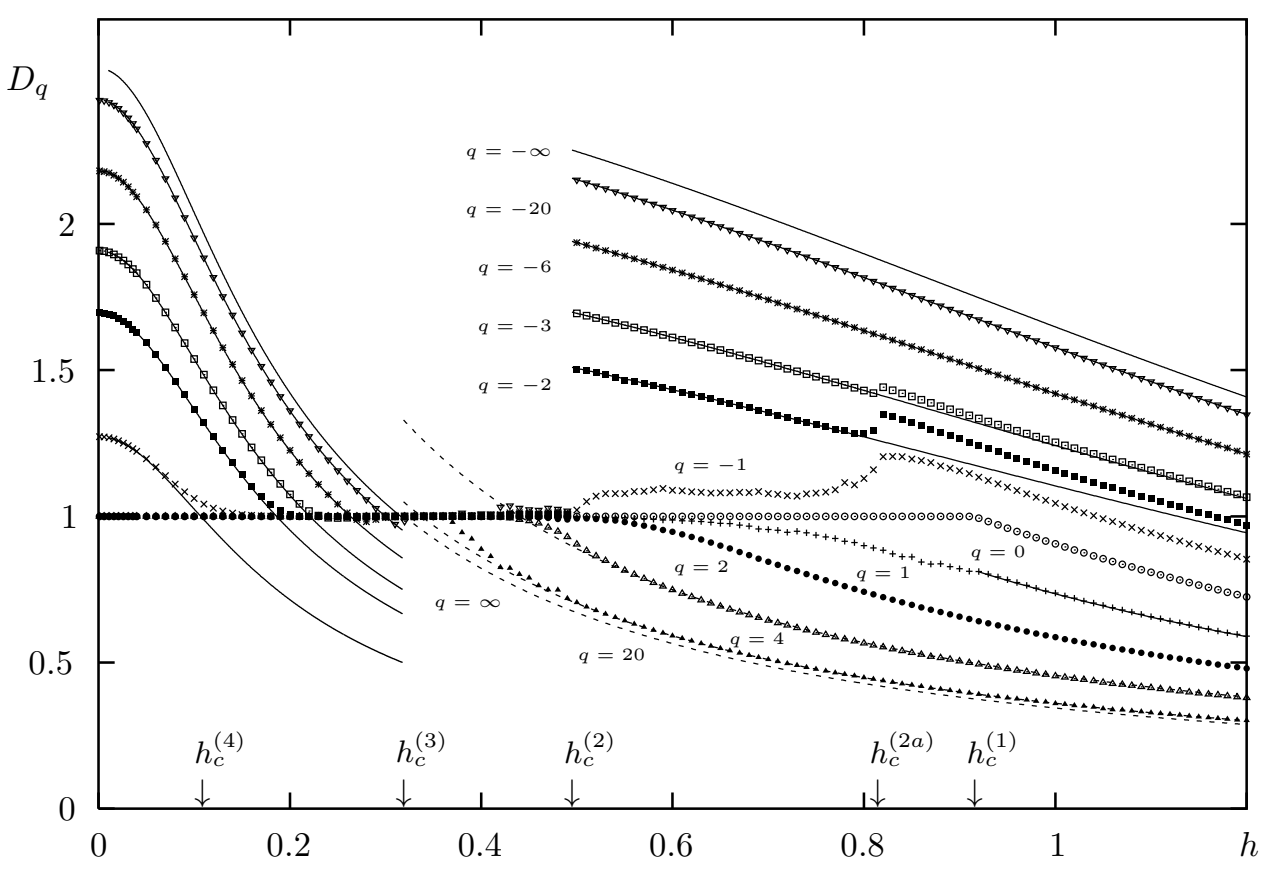

Figure 2. Generalized fractal dimensions $D_{q}$ of the invariant measure of the local effective field with $q=-20,-6,-3,-2,-1,0,1,2,4,20$ versus the amplitude of the local random field $h(\beta=1, J=1)$. The results were computed in the thermodynamic formalism using the new natural partition introduced by Behn and Lange 13. The significance of the critical values $h_{c}^{(n)}, n=1, \ldots, 4$, and $h_{c}^{(2 a)}$ is explained in the text. The solid and dashed lines are exact lower and upper bounds on $D_{q}$ respectively which are obtained in section 3 except for the solid line coinciding with the values of $D_{1}$ for $h>h_{c}^{(1)}$ which was obtained from (30).

$f_{ \pm}^{\prime}\left(x_{ \pm}^{*}\right)<\frac{1}{2}$ and converges to zero for $f_{ \pm}^{\prime}\left(x_{ \pm}^{*}\right)>\frac{1}{2}$ leading to the critical value 13

$$
h_{c}^{(3)}=\frac{1}{\beta} \operatorname{arsinh}\left(2^{-\frac{3}{2}}\left(1-9 e^{-4 \beta J}\right)^{\frac{1}{2}}\right) .
$$

The transition is again visible in numerically generated $p_{n}$ [13] as well as in the $D_{q^{-}}$ spectrum as the value of $h$ for which $D_{-\infty}$ begins to grow again for decreasing $h$, cf. figure 2. As was shown in [29], the generalized fractal dimension $D_{-\infty}$ has the value $D_{-\infty}=1$ at this point and $D_{-\infty}>1$ for $h<h_{c}^{(3)}$.

The last of the transitions which are already well understood occurs at $h_{c}^{(4)} \leq h_{c}^{(3)}$ when the slope of the coarse grained invariant measure density at $x_{ \pm}^{*}$ jumps from $\mp \infty$ to 0 . The condition for this is $f_{\sigma}^{\prime}\left(x_{\sigma}^{*}\right)=2^{-1 / 2}$ [14] resulting in [13]

$$
h_{c}^{(4)}=\frac{1}{\beta} \operatorname{arsinh}\left(3 \cdot 2^{-\frac{5}{2}}-\frac{1}{2}-\left(3 \cdot 2^{-\frac{5}{2}}+\frac{1}{2}\right) e^{-4 \beta J}\right)^{\frac{1}{2}} .
$$

The transition is visible in numerically generated densities $p_{n}$ [13] but not in the $D_{q^{-}}$ spectrum, cf. figure 2. Again, $D_{-\infty}$ can be calculated analytically and takes the value 

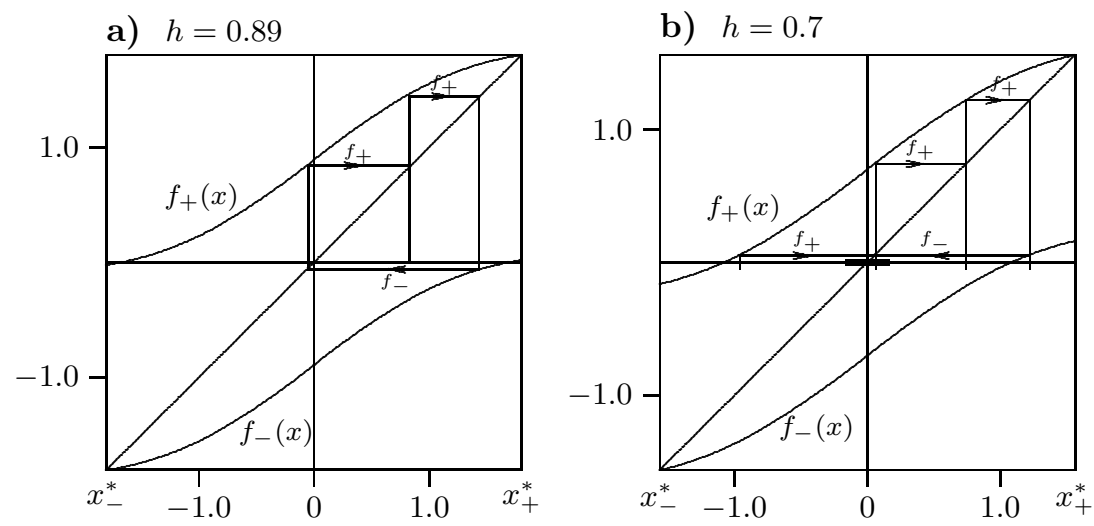

Figure 3. Orbits of period three for different values of $h$. (a) shows the case where no point of the orbit falls into the overlap region whereas in (b) the point $x_{\{-++\}}^{*}$ is in the overlap region and has therefore two predecessors $(\beta=1, J=1)$.

$D_{-\infty}=2$. In fact, $D_{-\infty}$ can be calculated analytically for all $h<h_{c}^{(3)}$ by considering that the scaling at the boundary in this case is weaker than at any other point 10$]$. This gives lower and upper bounds on $D_{q}$ which for $q \rightarrow-\infty$ converge against each other, see section 3 .

Please note that the effects summarized in this subsection only depend on the measure at the boundary of its support and therefore are not strictly multifractal effects.

\section{Orbits and their contribution to the invariant measure}

The orbit to a given symbolic sequence $\{\sigma\}$ consists of all preimages $f_{\{\sigma\}_{n}}^{-1}\left(x_{\{\sigma\}}^{*}\right)$, $n \in \mathbb{N}_{0}$. In the case of a periodic sequence $\{\sigma\}=\left(\{\sigma\}_{n}\right)_{\infty}$ with finite period $n$, the orbit consists of the fixed points of the $n$ functions $f_{\pi\{\sigma\}_{n}}$ in which $\pi$ denotes a cyclic permutation. To denote periodic orbits we will write for simplicity $\{\sigma\}_{n}$ instead of $\left(\{\sigma\}_{n}\right)_{\infty}$ The fixed points $x_{\{\sigma\}_{n}}^{*}$ of the periodic orbits are dense in the support of $\mu_{\infty}$ [34]. Furthermore, any point of the support of $\mu_{\infty}$ is contained in at least countably infinitely many orbits.

\subsection{Singularity of periodic orbits}

For the case of the fixed points $x_{+}^{*}$ of $f_{ \pm}$it has been shown before that their singularity can be calculated explicitly [14, 15. Fixed points are 1 - orbits and we generalize this concept to periodic orbits of arbitrary period length.

Let $y_{i}:=f_{\sigma_{i}}^{-1} \circ f_{\sigma_{i-1}}^{-1} \circ \cdots \circ f_{\sigma_{1}}^{-1}\left(x_{\{\sigma\}_{n}}^{*}\right), i=1, \ldots, n$, be the points of the periodic $n$-orbit defined by $\{\sigma\}_{n}$. We then have $y_{n}=y_{0}=x_{\{\sigma\}_{n}}^{*}$ because $x_{\{\sigma\}_{n}}^{*}$ is the fixed point of $f_{\{\sigma\}_{n}}$ by definition. An example for a periodic 3-orbit is shown in figure 3 .

In case that no overlap exists or - if it exists - that no point $y_{i}$ is in the overlap, the predecessor of each $y_{i}$ with respect to the iteration of the Frobenius-Perron equation is uniquely determined to be $y_{i-1}$. There is therefore only one term in the FrobeniusPerron equation at all $y_{i}$. We now investigate the singularity (pointwise dimension) 
of $\mu_{\infty}$ at $x_{\{\sigma\}_{n}}^{*}$. We assume that the scaling limit

$$
\lim _{\varepsilon \rightarrow 0} \frac{P_{\infty}\left(x_{\{\sigma\}_{n}}^{*}+\frac{\varepsilon}{2}\right)-P_{\infty}\left(x_{\{\sigma\}_{n}}^{*}-\frac{\varepsilon}{2}\right)}{\varepsilon^{\alpha_{\{\sigma\}_{n}}+1}}=: k
$$

exists for some finite $k \neq 0$ and some $\alpha_{\{\sigma\}_{n}} \in \mathbb{R}$. As is shown in Appendix A.1 this implies

$$
\lim _{\varepsilon \rightarrow 0} \frac{P_{\infty}\left(f_{\{\sigma\}_{n}}^{-1}\left(x_{\{\sigma\}_{n}}^{*}+\frac{\varepsilon}{2}\right)\right)-P\left(f_{\{\sigma\}_{n}}^{-1}\left(x_{\{\sigma\}_{n}}^{*}-\frac{\varepsilon}{2}\right)\right)}{\left(\left(f_{\{\sigma\}_{n}}^{-1}\right)^{\prime}\left(x_{\{\sigma\}_{n}}^{*}\right) \varepsilon\right)^{\alpha_{\{\sigma\}_{n}}+1}}=k .
$$

The $n$-fold iteration of the Frobenius-Perron equation yields

$$
\begin{aligned}
& P_{\infty}\left(x_{\{\sigma\}_{n}}^{*}+\frac{\varepsilon}{2}\right)-P_{\infty}\left(x_{\{\sigma\}_{n}}^{*}-\frac{\varepsilon}{2}\right) \\
& =\frac{1}{2^{n}}\left(P_{\infty}\left(f_{\{\sigma\}_{n}}^{-1}\left(x_{\{\sigma\}_{n}}^{*}+\frac{\varepsilon}{2}\right)\right)-P_{\infty}\left(f_{\{\sigma\}_{n}}^{-1}\left(x_{\{\sigma\}_{n}}^{*}-\frac{\varepsilon}{2}\right)\right)\right) .
\end{aligned}
$$

We denote the expression on the left hand side by $X$ and the one on the right hand side by $Y / 2^{n}$ and thus have $X / Y=1 / 2^{n}$. Inserting $1=k / k$ and using (12) and (13) with the introduced notation $X$ and $Y$ we get

$$
\lim _{\varepsilon \rightarrow 0}\left(\frac{X}{Y} \cdot \frac{Y /\left(\left(f_{\{\sigma\}_{n}}^{-1}\right)^{\prime}\left(x_{\{\sigma\}_{n}}^{*}\right) \varepsilon\right)^{\alpha_{\{\sigma\}_{n}+1}}}{X / \varepsilon^{\alpha_{\{\sigma\}_{n}}+1}}\right)=\frac{1}{2^{n}} .
$$

Most terms immediately cancel and we get

$$
\left(\left(f_{\{\sigma\}_{n}}^{-1}\right)^{\prime}\left(x_{\{\sigma\}_{n}}^{*}\right)\right)^{\alpha_{\{\sigma\}_{n}+1}}=2^{n} .
$$

Therefore

$$
\alpha_{\{\sigma\}_{n}}+1=\frac{n \ln 2}{\ln \left(\left(f_{\{\sigma\}_{n}}^{-1}\right)^{\prime}\left(x_{\{\sigma\}_{n}}^{*}\right)\right)}
$$

and with $\left(f_{\{\sigma\}_{n}}^{-1}\right)^{\prime}\left(x_{\{\sigma\}_{n}}^{*}\right)=\left(f_{\{\sigma\}_{n}}^{\prime}\left(f_{\{\sigma\}_{n}}^{-1}\left(x_{\{\sigma\}_{n}}^{*}\right)\right)\right)^{-1}=\left(f_{\{\sigma\}_{n}}^{\prime}\left(x_{\{\sigma\}_{n}}^{*}\right)\right)^{-1}$ we get

$$
\alpha_{\{\sigma\}_{n}}=-1-\frac{n \ln 2}{\ln \left(f_{\{\sigma\}_{n}}^{\prime}\left(x_{\{\sigma\}_{n}}^{*}\right)\right)} .
$$

This equation is invariant under cyclic permutation of $\{\sigma\}_{n}$ such that the scaling behaviour of the invariant measure at all $y_{i}, i=1, \ldots, n$, is given by the same Hölder exponent $\alpha_{\{\sigma\}_{n}}$. In other words, any point of the orbit has the same pointwise dimension $D_{p}=\alpha_{\{\sigma\}_{n}}-1$. We therefore call $\alpha_{\{\sigma\}_{n}}$ the singularity of the orbit $\{\sigma\}_{n}$. If $\alpha_{\{\sigma\}_{n}}<0$ the measure has a positive (i.e. strong) singularity at all $y_{i}$ and if $\alpha_{\{\sigma\}_{n}}>0$ the singularity is negative (i.e. weak).

As $f_{\{\sigma\}_{n}}^{\prime}\left(x_{\{\sigma\}_{n}}^{*}\right)=\prod_{i=1}^{n} f_{\sigma_{i}}^{\prime}\left(y_{i-1}\right)$, the derivatives $f_{\sigma_{i}}^{\prime}\left(y_{i-1}\right)=A^{\prime}\left(y_{i-1}\right)$ at the points $y_{i-1}$ of the periodic orbit determine the singularity $\alpha_{\{\sigma\}_{n}}$ and we finally have

$$
\alpha_{\{\sigma\}_{n}}=-1-\frac{n \ln 2}{\sum_{i=1}^{n} \ln A^{\prime}\left(y_{i}\right)} .
$$

$\S$ This assumption of strong scaling can be lifted in the non-overlapping case in which a generalization of (19) to arbitrary orbits can be proven provided the pointwise dimension of the fixed point exists, cf. subsection 2.2 . 


\begin{tabular}{|c|c|}
\hline head (finite) & tail (infinite) \\
\hline \multicolumn{2}{|l|}{ generic } \\
\hline $\begin{array}{l}+-+++--+- \\
\{\sigma\}_{n}\end{array}$ & $\begin{array}{l}+-+++--+-++---+\ldots \\
\{\tilde{\sigma}\}\end{array}$ \\
\hline \multicolumn{2}{|l|}{ periodic (here period 3) } \\
\hline $\begin{array}{l}-++-++-++ \\
\{\sigma\}_{9}=(-++)_{3}\end{array}$ & $\begin{array}{l}-++-++-++-++-++\ldots \\
(-++)_{\infty}\end{array}$ \\
\hline \multicolumn{2}{|c|}{ offshoot (arbitrary head, periodic tail) } \\
\hline $\begin{array}{l}+-++---++ \\
\{\sigma\}_{n}\end{array}$ & $\begin{array}{l}-++-++-++-++-++\ldots \\
(-++)_{\infty}\end{array}$ \\
\hline $\begin{array}{l}\text { head determines the } \\
\text { interval } I_{\{\sigma\}_{n}} \ni x_{\{\sigma\}}^{*}\end{array}$ & tail determines scaling at $x_{\{\sigma\}}^{*}$ \\
\hline
\end{tabular}

Table 1. Illustration of the terminology of symbolic sequences and the different roles played by head and tail.

A short calculation using the Frobenius-Perron equation (5) shows that the singularities of arbitrary points $x$ and $f_{\sigma}(x)$ are the same provided $f_{\sigma}(x)$ is not in the overlap, cf. Appendix A.2. The argument can be iterated such that the measure has the same singularity at any $x$ and all its images $f_{\{\tilde{\sigma}\}_{m}}(x)$ for any $\{\tilde{\sigma}\}$ for which no point $f_{\{\tilde{\sigma}\}_{i}}(x)$, $i=1, \ldots m$ is in $O$. Therefore not only the singularities at all points of a periodic orbit are the same but also the singularities at all points of non-periodic orbits in case the orbit also does not touch the overlap $O$. For orbits of the form $\{\tilde{\sigma}\}_{m}\left(\{\sigma\}_{n}\right)_{\infty}$ we know that this singularity is the singularity of $\{\sigma\}_{n}$, the periodic tail. We call non-periodic orbits of this type offshoots of the corresponding periodic orbit. The roles played by the head and the tail of a symbolic sequence $\{\sigma\}$ are summarized in table 1. Note that the choice of the length of the head is arbitrary, in a sense. Similar structures have been considered in [23].

\subsection{Singularity of generic orbits in the non-overlapping case}

In the last subsection we have seen that the fact that if the predecessor of each point of an orbit is unique we can explicitly calculate the singularity of the orbit as a function of the derivative of $A$ at the points of the orbit. In the non-overlapping case, $O=\emptyset$, the uniqueness of predecessors holds for any point in supp $\mu_{\infty}$. It is therefore natural to try to extend (19) to generic non-periodic orbits.

As a first step it is not difficult to show that if

$$
\lim _{n \rightarrow \infty} \frac{1}{n} \sum_{i=1}^{n} \ln A^{\prime}\left(x_{i}^{(n)}\right)
$$

exists for one choice of $x_{i}^{(n)} \in I_{\{\sigma\}_{i}}$ for a given $\{\sigma\}$, then it exists for any such choice and is independent of the particular choice made, cf. Appendix B. 
Let $x \in \operatorname{supp} \mu_{\infty}$ be such that 20] exists for the corresponding symbolic sequence $\{\sigma\}$. To calculate the limit $\varepsilon \rightarrow 0$ in (8) it is sufficient to consider $\varepsilon_{n}:=\left|I_{\{\sigma\}_{n}}\right|$ as $\frac{\varepsilon_{n+1}}{\varepsilon_{n}} \geq \min \left\{A^{\prime}(x) \mid x \in I\right\}=A^{\prime}\left(x_{+}^{*}\right)>0$ holds. We know from the general properties that $x=x_{\{\sigma\}}^{*} \in I_{\{\sigma\}_{n}}$ for all $n \in \mathbb{N}$. Because of the choice of $\varepsilon_{n}$ we have $B_{\varepsilon_{n}}(x) \supset I_{\{\sigma\}_{n}}$ such that $\mu_{\infty}\left(B_{\varepsilon_{n}}\right) \geq \mu_{\infty}\left(I_{\{\sigma\}_{n}}\right)=\frac{1}{2^{n}}$ leading to

$$
\frac{\ln \mu_{\infty}\left(B_{\varepsilon_{n}}\right)}{\ln \varepsilon_{n}} \leq \frac{-n \ln 2}{\ln \varepsilon_{n}} .
$$

On the other hand we also can choose $\varepsilon_{n}^{\prime}:=\left|f_{\{\sigma\}_{n}}(\Delta)\right|$. The interval $I_{\{\sigma\}_{n}}$ is neighboured by two gaps. One of the neighbouring gaps is always $f_{\{\sigma\}_{n-1}}(\Delta)$, the other is either $f_{\{\sigma\}_{m}}(\Delta)$ with $m<n-1$ or it is the complement of $I$. By contractivity of the RIFS we have $\left|f_{\{\sigma\}_{m}}(\Delta)\right|>\left|f_{\{\sigma\}_{n}}(\Delta)\right|$ for $m<n$, such that in either case the smallest gap neighbouring $I_{\{\sigma\}_{n}}$ is $f_{\{\sigma\}_{n-1}}(\Delta)$. This implies $\mu_{\infty}\left(B_{\varepsilon_{n}^{\prime}}\right) \leq \mu_{\infty}\left(I_{\{\sigma\}_{n}}\right)=\frac{1}{2^{n}}$ because $B_{\varepsilon_{n}^{\prime}}$ can not bridge any of the neighbouring gaps and thus only intersects $I_{\{\sigma\}_{n}}$. Therefore,

$$
\frac{\ln \mu_{\infty}\left(B_{\varepsilon_{n}^{\prime}}\right)}{\ln \varepsilon_{n}^{\prime}} \geq \frac{-n \ln 2}{\ln \varepsilon_{n}^{\prime}}
$$

Using the mean value theorem for $f_{\{\sigma\}_{n}}$ we obtain

$$
\begin{aligned}
\varepsilon_{n} & =\left|I_{\{\sigma\}_{n}}\right|=f_{\{\sigma\}_{n}}\left(x_{+}^{*}\right)-f_{\{\sigma\}_{n}}\left(x_{-}^{*}\right) \\
& =\left(f_{\{\sigma\}_{n}}\right)^{\prime}\left(x_{0}^{(n)}\right)\left(x_{+}^{*}-x_{-}^{*}\right)=\prod_{i=0}^{n-1} A^{\prime}\left(f_{\{\sigma\}_{i}}\left(x_{0}^{(n)}\right)\right)|I|
\end{aligned}
$$

for some $x_{0}^{(n)} \in I$. In the same fashion we get

$$
\varepsilon_{n}^{\prime}=\prod_{i=0}^{n-1} A^{\prime}\left(f_{\{\sigma\}_{i}}\left(x_{0}^{\prime(n)}\right)\right)|\Delta|
$$

for some other $x_{0}^{\prime(n)} \in I$. Taking (21) and (24) we obtain

$$
\begin{aligned}
\limsup _{n \rightarrow \infty} \frac{\ln \mu_{\infty}\left(B_{\varepsilon_{n}}\right)}{\ln \varepsilon_{n}} & \leq \limsup _{n \rightarrow \infty} \frac{-\ln 2}{\frac{1}{n} \sum_{i=0}^{n-1} \ln A^{\prime}\left(f_{\{\sigma\}_{i}}\left(x_{0}^{(n)}\right)\right)+\frac{1}{n} \ln |I|} \\
& =\lim _{n \rightarrow \infty} \frac{-n \ln 2}{\sum_{i=1}^{n} \ln A^{\prime}\left(f_{\{\sigma\}_{i}}^{-1}\left(x_{\{\sigma\}}^{*}\right)\right)}
\end{aligned}
$$

while using (22) and (25) yields

$$
\begin{aligned}
\liminf _{n \rightarrow \infty} \frac{\ln \mu_{\infty}\left(B_{\varepsilon_{n}^{\prime}}\right)}{\ln \varepsilon_{n}^{\prime}} & \geq \liminf _{n \rightarrow \infty} \frac{-\ln 2}{\frac{1}{n} \sum_{i=0}^{n-1} \ln A^{\prime}\left(f_{\{\sigma\}_{i}}\left(x_{0}^{\prime(n)}\right)\right)+\frac{1}{n} \ln |\Delta|} \\
& =\lim _{n \rightarrow \infty} \frac{-n \ln 2}{\sum_{i=1}^{n} \ln A^{\prime}\left(f_{\{\sigma\}_{i}}^{-1}\left(x_{\{\sigma\}}^{*}\right)\right)} .
\end{aligned}
$$

In both (27) and (29) the existence of the limit in (20) and the independence of (20) of the points $x_{i}^{(n)} \in I_{\{\sigma\}_{i}}$ was used to replace lim inf and lim sup by lim and to substitute 
the points of the orbit $\{\sigma\}$ for $x_{0}^{(n)}$ and $x_{0}^{\prime(n)}$ respectively. From (27) and (29) we immediately get that $D_{p}$ exists and is given by

$$
D_{p}=\lim _{n \rightarrow \infty} \frac{-n \ln 2}{\sum_{i=1}^{n} \ln A^{\prime}\left(f_{\{\sigma\}_{i}}^{-1}\left(x_{\{\sigma\}}^{*}\right)\right)} .
$$

Elton's ergodic theorem [35] implies that our assumption of the existence of (20) holds for almost all $\{\sigma\}$ which corresponds to $\mu_{\infty}$ - almost sure existence of the pointwise dimension $D_{p}(x)$. Elton's theorem together with (30) further implies that for $\mu_{\infty}-$ almost all $x$ the pointwise dimension takes the common value

$$
D_{p}(x) \stackrel{\mu_{\infty} \text {-a.s. }}{=} \frac{-\ln 2}{\int\left(\ln A^{\prime}(\xi)\right) \mu_{\infty}(d \xi)}=: \bar{D}_{p} .
$$

This result has direct consequences for the information dimension $D_{1}$. Proposition 2.1. in 36 implies that

$$
D_{H}\left(\left\{x \in I: D_{p}(x)=\bar{D}_{p}\right\}\right)=\bar{D}_{p}
$$

in which $D_{H}$ denotes the Hausdorff-dimension. General properties of the multifractal $f(\alpha)$-spectrum imply that $\alpha_{1}$ is the only fixed point of $f(\alpha)$ and that $\alpha_{1}=D_{1}$, cf. [37]. Therefore (32) implies $D_{1}=\bar{D}_{p}$. This fact is illustrated by the solid line in figure 2 coinciding with the numerically obtained values of $D_{1}$ for $h>h_{c}^{(1)}$ which was obtained through calculation of (31) using Edalat's $R$-integration [38]. (It also is a simple exercise to check on a computer that using the first $10^{5}$ digits of the dual representation of $\pi$ or $e$ as a symbolic sequence, the value of $\alpha$ obtained from (30) is also exactly the value $D_{1}-1$ in figure 2. Of course, the use of a random number generator instead of $\pi$ or $e$ yields the same result with probability one.)

Please note that the restriction to almost all $\{\sigma\}$ in the above is necessary as the sum in (20) does not converge for all $\{\sigma\}$. A simple example in which it does not converge is a sequence of bulks of plus and minus signs of ever increasing length. The length of the bulks can be chosen such that the sum in (20) keeps oscillating for any size of $n$.

\subsection{Singularity of orbits in the overlapping case}

In the previous subsections the condition that no point of the orbits under consideration is in the overlap $O$ was essential for the calculation of their singularity. In this section we investigate how the singularity of orbits is affected by the overlap $O$. Tuning the parameter $h$ changes $O$ as well as the location of the orbits. If (at least) one point $x$ of a periodic orbit is in $O$ this point has two predecessors and there are two terms in the Frobenius-Perron equation (5) at this $x$. The two terms contribute singularities $\alpha_{1}$ and $\alpha_{2}$, i.e. $\mu_{1}:=\mu_{\infty}\left(B_{\varepsilon}\left(f_{-}^{-1}(x)\right)\right) \sim \varepsilon^{\alpha_{1}}$ and $\mu_{2}:=\mu_{\infty}\left(B_{\varepsilon}\left(f_{+}^{-1}(x)\right)\right) \sim \varepsilon^{\alpha_{2}}$. Therefore, the singularity at $x$ will be $\inf \left\{\alpha_{1}, \alpha_{2}\right\}$, since $\mu_{\infty}\left(B_{\varepsilon}(x)\right) \sim \mu_{1}+\mu_{2} \sim \varepsilon^{\inf \left\{\alpha_{1}, \alpha_{2}\right\}}$. The mechanism is illustrated in figure 1 using the example of an orbit which will be important in the next subsection. In the case that the original singularity is stronger than or equal to the additionally contributed one, there are no consequences. In the case that the singularity is rather weak though, a weak singularity is replaced by a stronger singularity. In fact, the new singularity at the maximal value of $h$ for which $x$ is in $O$ is always rather strong as it stems from $x_{ \pm}^{*}$ where $A^{\prime}$ is small. The change 


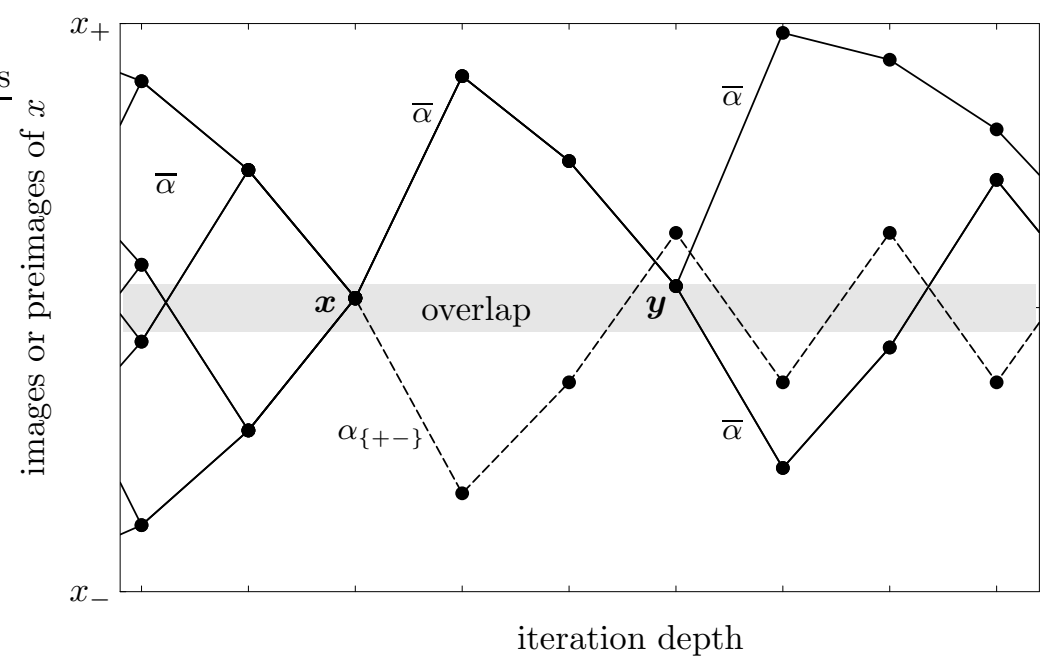

Figure 4. This figure illustrates the intricate structure of orbits and how weak singularities are superseded by stronger ones. The point $\left.x=x_{\{+--(+-)}^{*}\right)_{\infty}$ is in the overlap at $\beta=1, J=1, h=0.74\left(h_{c}^{(2)}<h<h_{c}^{(2 a)}\right)$. In this figure $x$, all its predecessors up to 6-fold application of $f_{ \pm}^{-1}$ and all its successors up to 2 -fold application of $f_{ \pm}$are shown. The points are the predecessors/successors whereas the connecting lines illustrate which point is mapped onto which. Note that the backward trajectory of $x$ branches each time a point is in the overlap whereas the predecessor is unique if the overlap is not touched. The dashed line connects points of the orbit $\left\{+--(+-)_{\infty}\right\}$ which is an offshoot of the $\{+-\}$ orbit and therefore has the singularity $\alpha_{\{+-\}} \approx 0.982$. The solid lines connect points of two other orbits which meet at $y \in O$, both carrying the generic singularity $\bar{\alpha}=\bar{D}_{p}-1 \approx-0.057$. At $x$ the weaker singularity $\alpha_{\{+-\}}$is superseded by $\bar{\alpha}$. The offshoots emerging from $x$ all have the stronger singularity $\bar{\alpha}$.

in the singularity may have a major impact on the $D_{q}$-spectrum especially if some or all of the weak but somewhat stronger singularities have already vanished.

A special role in the mechanism described above is played by the 1-orbits $\{+\}$ and $\{-\}$, because they never touch $O$, and the 2-orbit $\{+-\}$. At moderate overlap (small $|O|$ ) we have the situation illustrated in figure 5 . Since $x_{\{+-}^{*}$ is mapped to $x_{\{-+\}}^{*}$ and vice versa and because $f_{\sigma}$ is monotone, all points to the right of $x_{\{+-\}}^{*}$ are mapped to the right of $x_{\{-+\}}^{*}$ and all points to left of $x_{\{-+\}}^{*}$ are mapped to points left of $x_{\{+-\}}^{*}$. Therefore, any periodic $n$-orbit with $n>2$ must have at least one point inside $\left[x_{\{-+\}}^{*}, x_{\{+-\}}^{*}\right]$. Hence, the $\{+-\}$ orbit is the last periodic orbit to be reached by $O$.

The $\{+-\}$ orbit and its offshoots carry a very weak singularity as the $\{+-\}$ orbit always stays in regions with comparably large $A^{\prime}$, cf. equation (19). For $\beta$ and $J$ in the vicinity of $\beta=J=1$ one can show that the orbit $\{+-\}$ has even the weakest singularity of all periodic orbits [30]. Because of its weak singularity and the fact that all other periodic orbits and their offshoots are reached by $O$ before $x_{\{+-\}}^{*}$ is reached, the $\{+-\}$ orbit and its offshoots practically solely determine all $D_{q}$ with $q<0$ if $h$ is such that $O$ has nearly reached $x_{\{+-\}}^{*}$. If $O$ includes $x_{\{+-\}}^{*}$, i.e. $f_{-}\left(x_{+}^{*}\right) \geq x_{\{+-\}}^{*}$, $x_{\{+-\}}^{*}$ has additionally to the preimage $f_{+}^{-1}\left(x_{\{+-\}}^{*}\right)$ the preimage $f_{-}^{-1}\left(x_{\{+-\}}^{*}\right)$. (The same applies to $x_{\{-+\}}^{*}$, of course.) The additional preimages contribute a stronger 


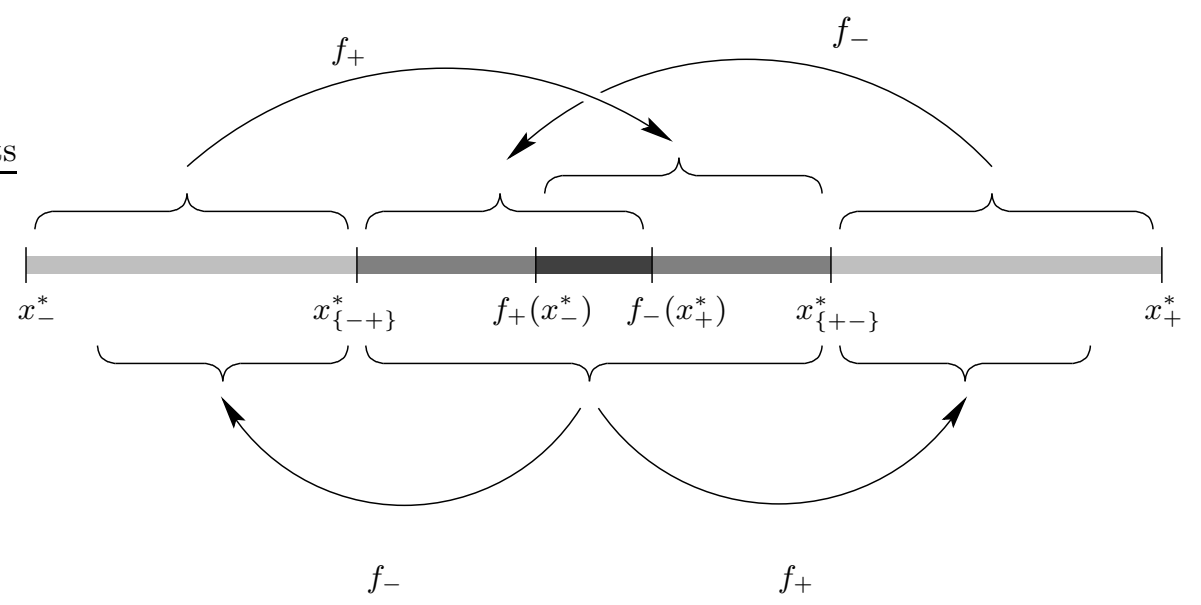

Figure 5. Mapping of subintervals of $I=\left[x_{-}^{*}, x_{+}^{*}\right]$ under $f_{+}$and $f_{-}$elucidating the importance of the interval $\left[x_{\{-+\}}^{*}, x_{\{+-\}}^{*}\right]$. The latter is mapped onto parts of the outer intervals $\left[x_{-}^{*}, x_{\{-+\}}^{*}\right]$ and $\left[x_{\{+-\}}^{*}, x_{+}^{*}\right]$ under $f_{-}$and $f_{+}$while these outer intervals are itself preimages of parts of $\left[x_{\{-+\}}^{*}, x_{\{+-\}}^{*}\right]$. The points in the overlap $\left[f_{+}\left(x_{-}^{*}\right), f_{-}\left(x_{+}^{*}\right)\right]$ have two predecessors, one stemming from the left under $f_{+}$and one from the right under $f_{-}$(all in the case of moderate overlap).

singularity than the original weak singularity of the $\{+-\}$ orbit. Thus, the weak singularity of the $\{+-\}$ orbit and its offshoots is superseded and, as all other periodic orbits have been reached by $O$ before, all $D_{q}$ with negative $q$ have collapsed to $D_{q}=1$ at this point. The critical value $h_{c}^{(2)}$ at which the collapse takes place is therefore given by the condition

$$
f_{-}\left(x_{+}^{*}\right)=x_{\{+-\}}^{*} \cdot
$$

So far we have only discussed periodic orbits and their offshoots. Other nonperiodic orbits do not play a major role because they generically have the rather strong singularity $\bar{\alpha}=\bar{D}_{p}-1=D_{1}-1$ and also generically have points inside $\left[x_{\{-+\}}^{*}, x_{\{+-\}}^{*}\right]$ such that they are reached by $O$ before the $\{+-\}$ orbit is reached.

After the collapse the right part of the $f(\alpha)$-spectrum of the invariant measure has vanished since the weaker negative singularities have all been superseded by stronger ones, cf. figure 6a. A similar collapse of parts of the multifractal spectrum has previously been observed in the superposition of equal-scale [39] and multi-scale [40, 41] Cantor sets showing that effects of this type appear in a wide variety of applications.

Let us now determine $h_{c}^{(2)}$ explicitly. In a first step we need explicit expressions for $x_{+}^{*}=-x_{-}^{*}$ and $x_{\{+-\}}^{*}=-x_{\{-+\}}^{*}$. The fixed point $x_{+}^{*}$ is defined by $f_{+}\left(x_{+}^{*}\right)=x_{+}^{*}$. With the notation $z=e^{2 \beta x_{+}^{*}}$ this yields the equation

$$
z^{2}-\left(e^{2 \beta J}\left(e^{2 \beta h}-1\right)\right) z-e^{2 \beta h}=0
$$

with the solution

$$
x_{+}^{*}=\frac{1}{2 \beta} \ln \left(K+\sqrt{K^{2}+e^{2 \beta h}}\right)
$$


a)

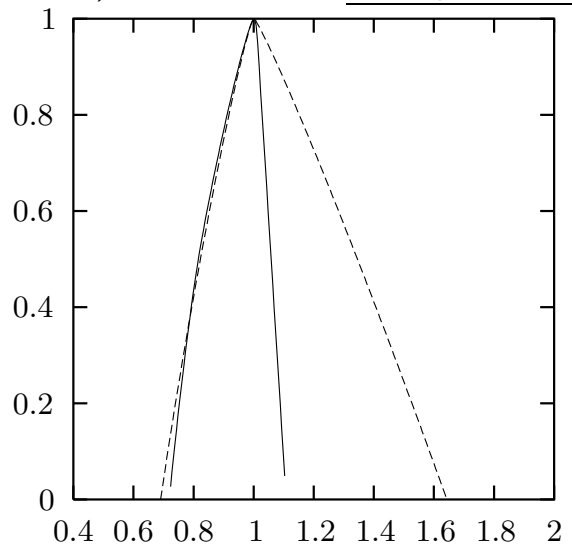

b)

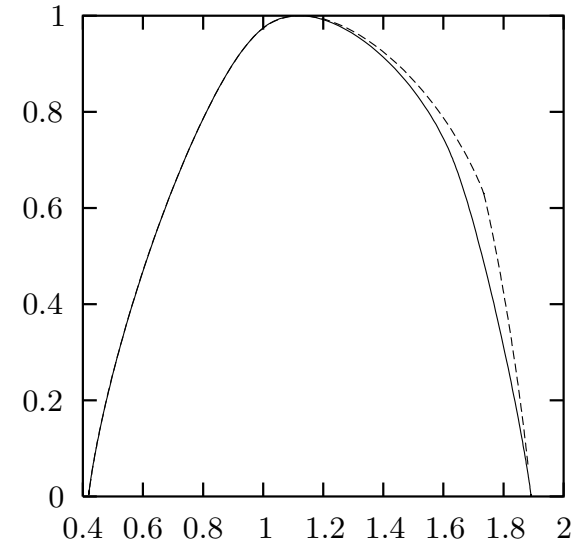

Figure 6. Collapse of the right part of the $f(\alpha)$-spectrum at $h_{c}^{(2)}$ and $h_{c}^{(2 a)}$. In (a) the spectra at $h=0.49385>h_{c}^{(2)}$ (dashed line) and $h=0.4938<h_{c}^{(2)}$ (solid line) are shown. In (b) the spectra are at $h=0.8136>h_{c}^{(2 a)}$ (dashed line) and $h=0.8128<h_{c}^{(2 a)}$ (solid line). The spectra were obtained by a numerical Legendre transform of the corresponding $D_{q}$-spectra. These were generated with the same algorithm as in figure 8 with a recursion depth of $21(\beta=1, J=1)$.

where $K=e^{2 \beta J}\left(e^{2 \beta h}-1\right) / 2$. To obtain $x_{\{+-\}}^{*}$ we exploit $x_{\{+-\}}^{*}=-x_{\{-+\}}^{*}=$ $-f_{-}\left(x_{\{+-\}}^{*}\right)$. With $z=e^{2 \beta x_{\{+-\}}^{*}}$ this yields

$$
z^{2}+e^{-2 \beta J}\left(e^{2 \beta h}-1\right) z-e^{2 \beta h}=0
$$

and therefore

$$
x_{\{+-\}}^{*}=\frac{1}{2 \beta} \ln \left(\tilde{K}+\sqrt{\tilde{K}^{2}+e^{2 \beta h}}\right)
$$

with $\tilde{K}=e^{-2 \beta J}\left(e^{2 \beta h}-1\right) / 2$.

The equation $f_{-}\left(x_{+}^{*}\right)=x_{\{+-\}}^{*}$ is equivalent to $x_{+}^{*}=x_{\{+-\}}^{*}+2 h$. With the explicit expressions for $x_{+}^{*}$ and $x_{\{+-\}}^{*}$ we get

$$
2\left(\cosh \left(2 \beta h_{c}^{(2)}\right)\right)^{3}+3\left(\cosh \left(2 \beta h_{c}^{(2)}\right)\right)^{2}=(\cosh (2 \beta J))^{2} .
$$

This equation has exactly one real solution for $\cosh \left(2 \beta h_{c}^{(2)}\right)$ resulting in

$$
h_{c}^{(2)}=\frac{1}{2 \beta} \operatorname{arcosh}\left(\cosh \left(\frac{4}{3} \beta J\right)-\frac{1}{2}\right) .
$$

The phase diagram for the transition at $h_{c}^{(2)}$ is shown in figure 7. One clearly sees that there is a critical line $\beta(J)$ such that there is no phase transition possible for all $\beta<\beta(J)$. This line is given by the condition $h_{c}^{(2)}=0$ corresponding to

$$
\beta(J)=\frac{3}{4 J} \operatorname{arcosh}\left(\frac{3}{2}\right)=\frac{1}{2 J} \ln (2+\sqrt{5}) .
$$

With (39) and (40) we thus have a complete analytical understanding of the occurrence and the position of the transition at $h_{c}^{(2)}$. 


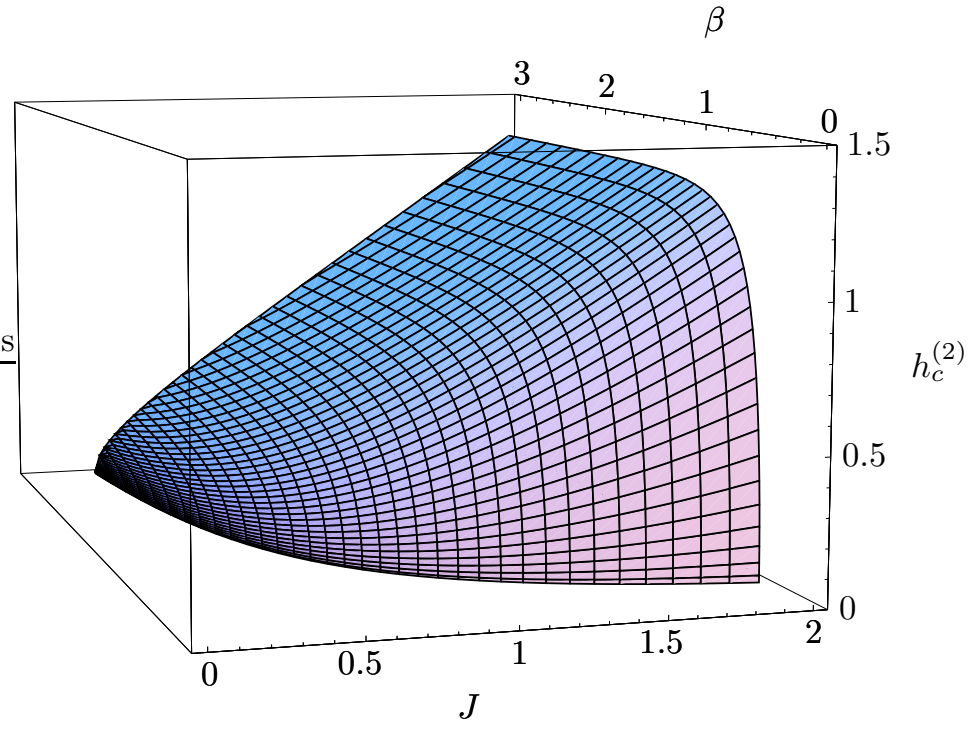

Figure 7. Surface plot of the critical value $h_{c}^{(2)}$ against $\beta$ and $J$. The plot was obtained from (39).

\subsection{Transition of the measure at $h_{c}^{(2 a)}$}

We have seen in the last subsection that the sharp drop of all $D_{q}$ with negative $q$ at $h_{c}^{(2)}$ can be explained through the analysis of the properties of the $\{+-\}$ orbit which is on the one hand an orbit with very weak singularity and on the other hand the last periodic orbit (with decreasing $h$ ) to be affected by th overlap $O$. To understand the smaller drop at $h_{c}^{(2 a)}$ it is therefore natural to look for periodic orbits with weak singularity which also are affected by the overlap relatively late. We will now argue that the family of periodic orbits of the form $\left\{+--(+-)_{n}\right\}, n \in \mathbb{N}$ fulfills these conditions and that their change in singularity indeed causes the transition at $h_{c}^{(2 a)}$. To present the precise argument we need to investigate some properties of this family of orbits.

For large $n$ most points of the $\left\{+--(+-)_{n}\right\}$ orbits are close to the points of the $\{+-\}$ orbit. Thus, having (19) in mind, the singularities of these orbits have a similar strength as singularity of the $\{+-\}$ orbit, i.e. they are weak, provided that no point of the orbits is in the overlap $O$. The larger $n$ the larger is the fraction of points of the corresponding orbit which are close to $x_{\{+-\}}^{*}$ and $x_{\{-+\}}^{*}$. Therefore, the singularities get weaker with growing $n$.

Concerning the position of the orbits $\left\{+--(+-)_{n}\right\}$ we first note that the points $z_{n}:=x_{\left\{+--(+-)_{n}\right\}}^{*}$ are for each of the orbits $\left\{+--(+-)_{n}\right\}$ the closest points to the overlap $O$. As $f_{\left\{+--(+-)_{n}\right\}}\left(x_{0}^{(n)}\right) \rightarrow x_{\left\{+--(+-)_{\infty}\right\}}^{*}$ for any choice of initial points $x_{0}^{(n)} \in I$ and $n \rightarrow \infty$, we get with $x_{0}^{(n)}:=z_{n}=x_{\left\{+--(+-)_{n}\right\}}^{*}$ that $z_{n} \rightarrow x_{\left\{+--(+-)_{\infty}\right\}}^{*}$ for $n \rightarrow \infty$. Furthermore, it is an easy exercise to check that $z_{n}$ is monotonously growing with $n$.

Taking these properties together we conclude that each of the periodic orbits of the form $\left\{+--(+-)_{n}\right\}$ is affected by the overlap as soon as $z_{n}$ is in $O$ and that the 


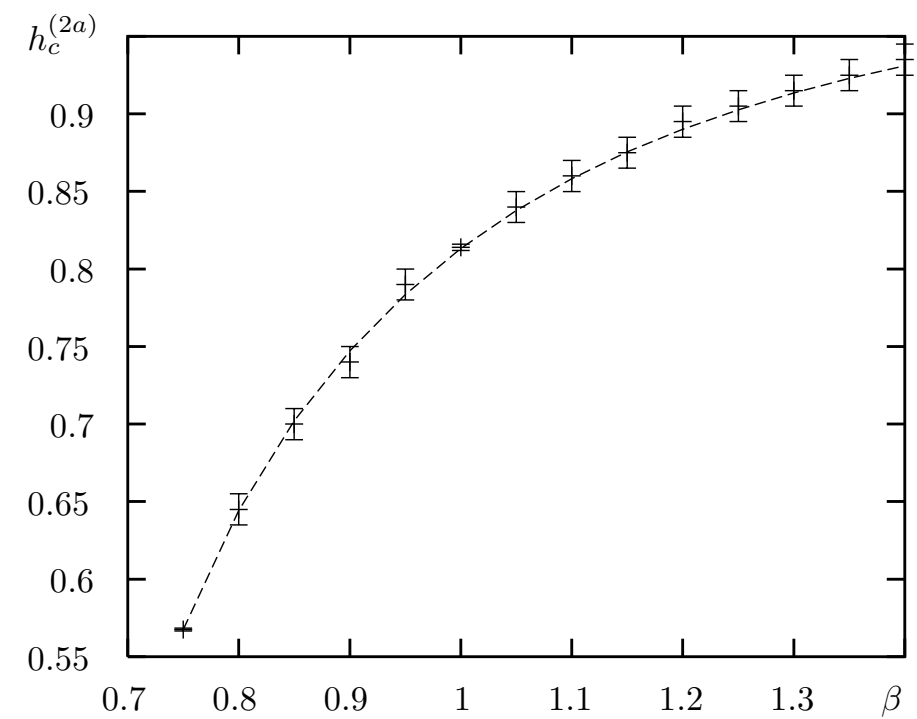

Figure 8. $h_{c}^{(2 a)}$ obtained from the analytical condition (41) (dashed line) compared with the location of the small drops in $D_{q}$ (error-bars), cf. figure 2 . The errors are estimated and mainly due to the errors in the determination of the location of the drops in the numerically obtained $D_{q}$-spectra $(J=1)$.

orbits with weaker singularity (those with large $n$ ) are affected by the overlap later than the orbits with somewhat stronger singularity. Finally, as the $z_{n}$ converge to $x_{\left\{+--(+-)_{\infty}\right\}}^{*}$, there are still countably infinitely many orbits $\left\{+--(+-)_{n}\right\}, n \geq N$ with some $N \in \mathbb{N}$ left which are not affected by the overlap as long as $x_{\left\{+--(+-)_{\infty}\right\}}^{*}$ is not in $O$. As soon as $x_{\left\{+--(+-)_{\infty}\right\}}^{*}$ gets into the overlap $O$, all orbits $\left\{+--(+-)_{n}\right\}$ are in the overlap and their singularity therefore superseded. The critical field strength $h_{c}^{(2 a)}$ is thus determined by the condition

$$
f_{-}\left(x_{+}^{*}\right)=x_{+--(+-)_{\infty}}^{*}=f_{\{+-\}}\left(x_{\{-+\}}^{*}\right) .
$$

This criterion is in perfect agreement with the numerically obtained positions of the

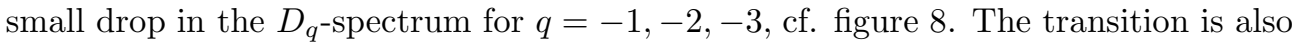
visible in the $f(\alpha)$-spectrum. For $h \rightarrow h_{c}^{(2 a)}+0$ a cusp develops and the spectrum collapses to a smooth form again at $h_{c}^{(2 a)}$, cf. figure $6 \mathrm{~b}$.

So far we only addressed the periodic orbits $\left\{+--(+-)_{n}\right\}$ neglecting their offshoots. Each such orbit has countably many offshoots. From these offshoots those originating from points left of $O$ and containing exclusively additional - as well as those originating from points to the right of $O$ and only containing additional + are also not affected by the overlap as long as the corresponding periodic orbit is not. Thus, at $h_{c}^{(2 a)}$ countably infinitely many periodic orbits with each countably many offshoots of the described form vanish at once. This explains why this transition is visible in the $D_{q}$-spectrum in contrast to events of single orbits (and their offshoots) being affected by the overlap at any value of $h$.

The fact that the transition is not visible in $D_{q}$ with large negative $q$ is also easily understood in this framework. As the singularities of all $\left\{+--(+-)_{n}\right\}$ orbits are 
a) $h=0.85>h_{c}^{(2 a)}$

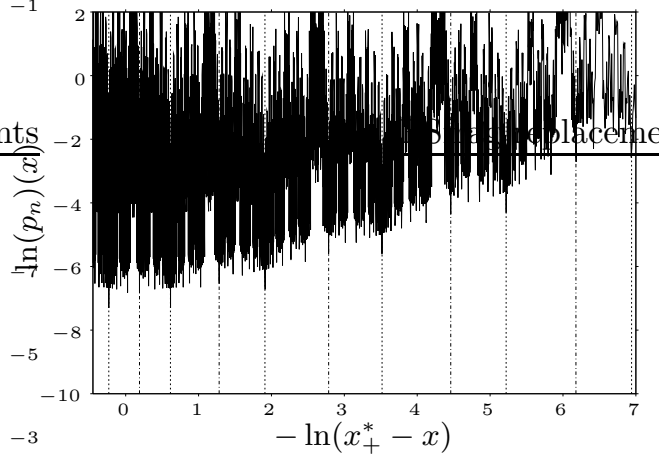

c) $h \approx 0.5436>h_{c}^{(2)}$

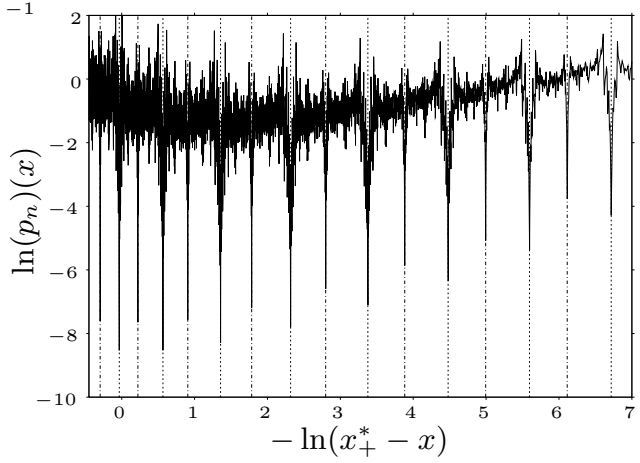

b) $h=0.7<h_{c}^{(2 a)}$

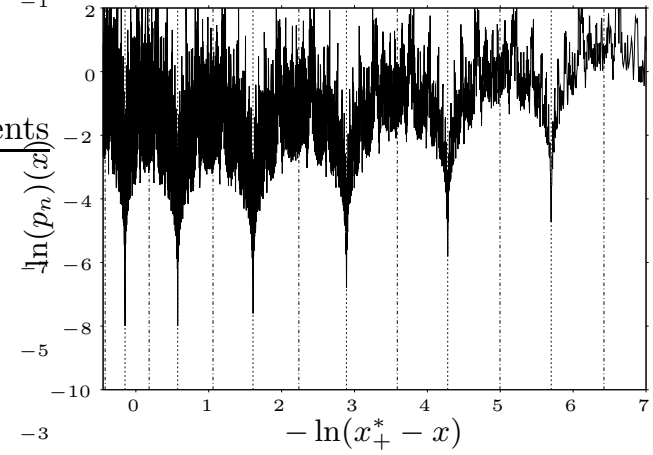

d) $h=0.48<h_{c}^{(2)}$

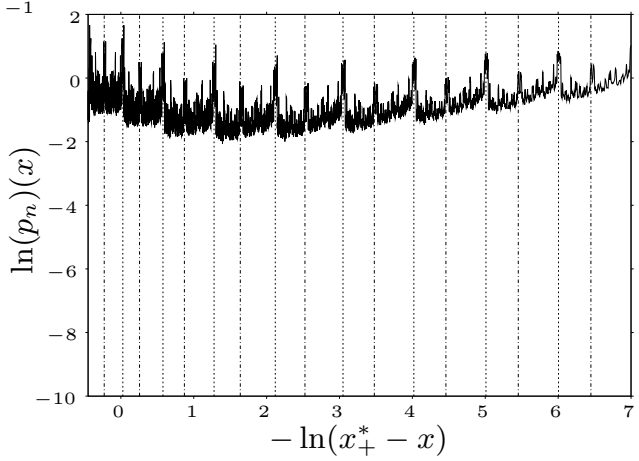

Figure 9. Double logarithmic plot of $p_{n}$ generated from the band structure after $n=21$ iterations. The negative peaks correspond to deep cuts in $p_{n}$. The dotted lines mark the position of $x_{\left\{(+)_{k}(+-)_{\infty}\right\}}^{*}$, i.e. the points of the $\{+-\}$ orbit and its offshoots, and the dash-dotted lines the positions of $x_{\left\{(+)_{k}+--(+-)_{\infty}\right\}}^{*}$, i.e. the points of the $\left\{+--(+-)_{\infty}\right\}$ orbit and its offshoots. In (a) for $h>h_{c}^{(2 a)}$ deep cuts are visible at all marked positions whereas in (b) for $h<h_{c}^{(2 a)}$ the deep cuts at $x_{\left\{(+)_{k}+--(+-)_{\infty}\right\}}$ have vanished. They reappear in (c) at a critical field strength $h \approx 0.5436$ as is discussed in the text whereas in (d) for $h<h_{c}^{(2)}$ all deep cuts have vanished $(\beta=1, J=1)$.

similar to but somewhat stronger than the singularity of the $\{+-\}$ orbit, the $\{+-\}$ orbit and its offshoots dominate $D_{q}$ for large negative $q$ and the transition is not visible.

Please note the symmetry of the system which allows the same reasoning with the 'opposite' orbits $\left\{-++(-+)_{n}\right\}$ resulting in an equivalent result.

Even though the effect is due to the periodic orbits $\left\{+--(+-)_{n}\right\}$ and their offshoots, the orbit entering into condition (411) is an offshoot of the $\{+-\}$ orbit, namely $\left\{+--(+-)_{\infty}\right\}$. This orbit thus seems to play a similar role as the $\{+-\}$ orbit for the transition at $h_{c}^{(2)}$. For illustration we have generated an approximation of $p_{n}$ and calculated the position of the points of the $\{+-\}$ orbit and its offshoots of the form $\left\{(+)_{n}(+-)_{\infty}\right\}$ (dotted lines in figure 9) as well as the position of the points of the $\left\{+--(+-)_{\infty}\right\}$ orbit and its offshoots of the form $\left\{(+)_{n}+--(+-)_{\infty}\right\}$ (dashed lines in figure 9). It is obvious that there is a bunch of weak singularities in the vicinity of $x_{\left\{+--(+-)_{n}\right\}}^{*}$ and in the vicinity of the points of the offshoots which all vanish when 


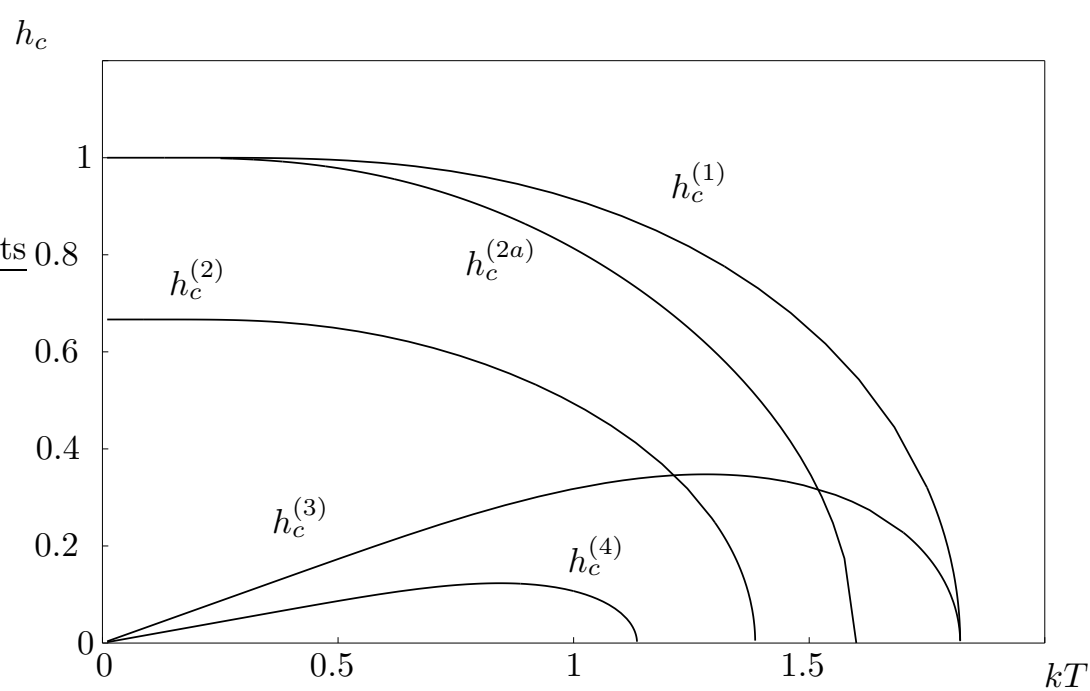

Figure 10. Phase diagram for the transitions of the invariant measure derived from the random field Ising model. The $h_{c}^{(n)}$ are the critical random field strengths defined in the text. The remarkable fact that the lines of $h_{c}^{(2)}$ and $h_{c}^{(3)}$ as well as the lines of $h_{c}^{(2 a)}$ and $h_{c}^{(3)}$ intersect shows that there is a variety of different scenarios depending on the choice of $k T$. For example at $k t=1.3$, the transition at $h_{c}^{(2)}$ precedes the one at $h_{c}^{(3)}$ while the transition at $h_{c}^{(4)}$ is non-existent $(J=1)$.

decreasing $h$ below $h_{c}^{(2 a)}$, cf. figure $9 \mathrm{a}$ and b.

The attentive reader will have noticed that we have argued that the weak singularity of an orbit which is touched by the overlap $O$ for some $h_{0}$ but not for $h>h_{0}$ will be superseded by the strong singularity of the $\{+\}$ or the $\{-\}$ orbit. For smaller $h$ in the generic case the additionally contributed singularity will be $D_{1}-1$ and thus also rather large. It is not excluded though that two weak singularities are combined resulting in a weak singularity even though the orbit is in the overlap. A prominent example of this effect is the value of $h$ for which $x_{\left\{+--(+-)_{\infty}\right\}}^{*}=x_{\left\{-++(-+)_{\infty}\right\}}^{*}=0$. At this value of $h$ new deep cuts in the approximated measure density appear at $x_{\left\{+--(+-)_{\infty}\right\}}^{*}$ and its offshoots, cf. figure $9 \mathrm{c}$. The effect on the $D_{q}$-spectrum is negligible though as this is a rare event only affecting a single orbit and its offshoots at a given $h$.

To summarize, the crucial feature for a visible transition in the $D_{q}$-spectrum is that a non-negligible fraction of orbits with weak singularities is affected by the overlap at one sharp critical value $h_{c}$ resulting in a drop of $D_{q}$ with negative $q$. It is not excluded that there are more transitions of this type which might become observable with further increase in numerical accuracy in the future. Our arguments hint to the conjecture that these transitions, if existent, should take place at $h>h_{c}^{(2 a)}$.

The formulae given in the last two subsections allow to draw a phase diagram for the phase transitions in the $D_{q}$-spectrum of the invariant measure which have been observed so far, cf. figure 10. It should be emphasized that these 'phase transitions' are not phase transitions of physical quantities like the (local) magnetisation or the Edwards-Anderson parameter. 


\section{Bounds on the $D_{q}$-spectrum}

Let in the following $\mu$ denote the invariant measure. As mentioned above there are natural bounds on $D_{q}$. They are induced by

$$
\mu_{(x)}^{q} \leq \sum_{i} \mu_{i}^{q}
$$

where $x$ is some point in the support of $\mu$ and $\mu_{(x)}$ is the measure of the box containing $x$. Let us now first consider the case $q<0$. In this case inequality (42) immediately yields

$$
D_{q} \geq \frac{q}{q-1} \lim _{\varepsilon \rightarrow 0} \frac{\ln \left(\mu_{(x)}\right)}{\ln \varepsilon} .
$$

The limit on the right hand side is the pointwise dimension at $x$. If we choose $x=x_{+}^{*}$ we can calculate the pointwise dimension at $x$ for any $h, T \neq 1, J$ using (19). This gives the lower bound

$$
D_{q} \geq \frac{q}{1-q} \frac{\ln 2}{\ln \left(A^{\prime}\left(x_{+}^{*}\right)\right)}
$$

In the region $h>h_{c}^{(2)}$ we also can calculate the pointwise dimension at $x_{\{+-\}}^{*}$. With $x=x_{\{+-\}}^{*}$ we get the bound

$$
D_{q} \geq \frac{q}{1-q} \frac{\ln 2}{\ln \left(A^{\prime}\left(x_{\{+-\}}^{*}\right)\right)} .
$$

These lower bounds are shown as solid lines in figure 2 .

Whenever we know that the invariant measure scales most weakly at some point $x$ of its support and we can calculate the pointwise dimension at $x$, we also can give an upper bound induced by

$$
\sum_{i} \mu_{i}^{q} \leq N \mu_{(x)}^{q}
$$

In this $N$ is the number of boxes and is essentially proportional to $1 / \varepsilon$. In the region $h<h_{c}^{(3)}$ the measure is assumed to scale most weakly at $x_{+}^{*}$ (and $x_{-}^{*}$ ) such that inserting (46) into (7) we obtain the upper bound

$$
D_{q} \leq \frac{1}{1-q}\left(1+\frac{q \ln 2}{\ln \left(A^{\prime}\left(x_{+}^{*}\right)\right)}\right)
$$

In the region $h>h_{c}^{(2)}$ the scaling is assumed to be weakest at $x_{\{+-\}}^{*}\left(\right.$ and $\left.x_{\{-+\}}^{*}\right)$ yielding

$$
D_{q} \leq \frac{1}{1-q}\left(1+\frac{q \ln 2}{\ln \left(A^{\prime}\left(x_{\{+-\}}^{*}\right)\right)}\right) .
$$

Obviously the upper and lower bounds converge against each other in both regions as $q \rightarrow-\infty$ such that we get the explicit expressions

$$
D_{-\infty}= \begin{cases}-\frac{\ln 2}{\ln \left(A^{\prime}\left(x_{+}^{*}\right)\right)} & h<h_{c}^{(3)} \\ -\frac{\ln 2}{\ln \left(A^{\prime}\left(x_{i+-\}}^{*}\right)\right)} & h>h_{c}^{(2)} .\end{cases}
$$


The expression for the region $h<h_{c}^{(3)}$ was already given in [29] whereas the expression for the region $h>h_{c}^{(2)}$ needs our analysis of the singularity of orbits in section 2.1. These upper bounds are in general not as good as the lower ones found above and are not shown in figure 2 .

In the case $q>0$ all considerations remain valid for the bounds based on (42) which yields by use of (19) the upper bounds

$$
D_{q} \leq \frac{q}{1-q} \frac{\ln 2}{\ln \left(A^{\prime}\left(x_{+}^{*}\right)\right)}
$$

for any $h$ and the sharper condition

$$
D_{q} \leq \frac{q}{1-q} \frac{\ln 2}{\ln \left(A^{\prime}\left(x_{\{+-\}}^{*}\right)\right)}
$$

for $h>h_{c}^{(2)}$. These upper bounds (51) are shown as dashed lines in figure 2.

Lower bounds on $D_{q}$ can be obtained by considering points $x$ at which the scaling is maximally strong. This is assumed to be true for $x_{+}^{*}$ and $x_{-}^{*}$ in the region $h>h_{c}^{(3)}$. Therefore we get

$$
D_{q} \geq \frac{1}{1-q}\left(1+\frac{q \ln 2}{\ln \left(A^{\prime}\left(x_{+}^{*}\right)\right)}\right)
$$

These lower bounds are not as good as the upper bounds (51) and are not shown in figure 2. In the region $h>h_{c}^{(2)}$ the lower and upper bounds for $D_{q}$ converge against a common value for $q \rightarrow \infty$ yielding

$$
D_{\infty}=-\frac{\ln 2}{\ln \left(A^{\prime}\left(x_{+}^{*}\right)\right)} .
$$

The expressions (49) and (53) generalize results priorly obtained [10] for $h>h_{c}^{(1)}$.

Note that for $q<0(q>0)$ the upper (lower) bounds rest on the assumption of minimal (maximal) scaling at $x_{+}^{*}\left(x_{-}^{*}\right)$ and $x_{\{+-\}}^{*}\left(x_{\{-+\}}^{*}\right)$ whereas the lower (upper) bounds do not need any additional assumptions. In figure 2 only the lower (upper) bounds are shown.

The fact that for $q \leq-2(q \geq 4)$ the lower (upper) bounds are more or less identical with the numerical data (cf. figure 2) shows that in these cases the generalized dimensions $D_{q}$ are solely determined by the scaling at certain points, i.e. at $x_{+}^{*}$ and $x_{-}^{*}$ for $h<h_{c}^{(3)}$ and at $x_{\{+-\}}^{*}$ for $h>h_{c}^{(2)}$. This reinforces our previous argument why the small drop in the $D_{q}$-spectrum at $h_{c}^{(2 a)}$ is not visible in numerical data for $q<-3$.

\section{Concluding remarks}

By generalizing arguments, hitherto mainly applied to fixed points, to orbits we have been able to calculate the singularity of all periodic orbits not touching the overlap $O$ and the generic ( $\mu$-a.s.) singularity of orbits in the non-overlapping case. We then investigated the effects of a non-void overlap on the singularity of orbits. While being a relevant result in its own right the knowledge of the singularity of orbits and their 


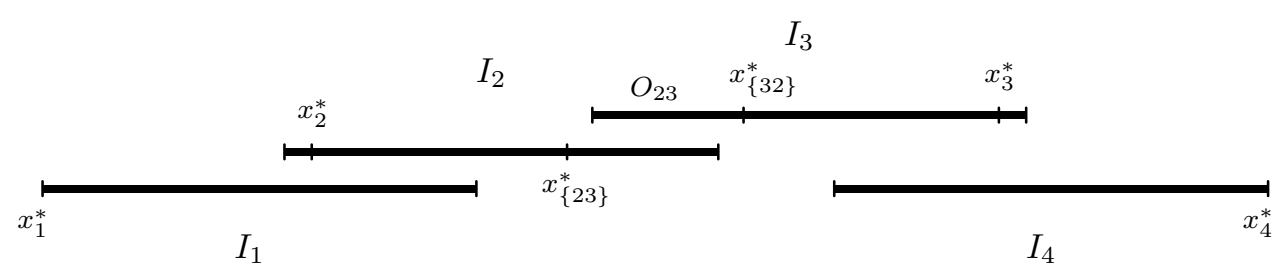

Figure 11. Sample situation of first order bands in the symmetric four Dirac mass case in the vicinity of a transition similar to the one at $h_{c}^{(2)}$ in the text. If, as shown in the figure, $H$ is large enough, $x_{\{23\}}^{*}$ and $x_{\{32\}}^{*}$ are not included in $I_{1}$ and $I_{2}$ respectively. In this case $x_{\{23\}}^{*}$ has the unique predecessor $x_{\{32\}}^{*}$ and vice versa exactly like $x_{\{-+\}}^{*}$ and $x_{\{+-\}}^{*}$ in the dichotomous case. The situation is therefore identical to the situation in the dichotomous case and the transition takes place at $h=h_{c}^{(2)} .(\beta=1, h=0.7, H=1.8)$

dependence on touching the overlap or not also provided the explanation for two phase transitions in the multifractal $D_{q}$-spectrum of the invariant measure.

RIFS with similar properties as the one discussed here also appear in a variety of other contexts [21, 22, 23, 29]. In 29] a similar phase transition in the $D_{q}$-spectrum already has been observed. The explanation of the mechanism causing such transitions does not crucially depend on the special choice of the RIFS we investigated here. Let us briefly discuss some obvious generalizations.

Dropping the restriction of symmetry of the random field distribution leads to the loss of various symmetries of the orbit structure, e.g. $x_{+}^{*} \neq-x_{-}^{*}$ and $x_{\{+-\}}^{*} \neq-x_{\{-+\}}^{*}$, and of the resulting calculational simplicity. The general orbit structure underlying our explanations of transitions in the $D_{q}$-spectrum however remains. Therefore, the transitions also exist in the non-symmetric case and can be analysed in the same way as above in the symmetric case. Some numerical results may be found [13]. The explicit calculations are far more complicated though.

Considering more complex random field distributions like e.g. $n$ (symmetrically or non-symmetrically distributed) Dirac masses considerably complicates the system. The mechanisms causing phase transitions in the $D_{q}$-spectra are nevertheless still the same. Let us briefly outline the example of four symmetrically distributed Dirac masses located at $\{-H,-h, h, H\}$ as the random field distribution, i.e. the RIFS $\left\{f_{1}, f_{2}, f_{3}, f_{4}\right\}=\{A-H, A-h, A+h, A+H\}$ with $h<H \in \mathbb{R}^{+}$:

- For any given $\beta$ a transition line in the $h-H$-plane exists between a region with $D_{0}=1$ and $D_{0}<1$ which is easily determined from the overlap conditions of the first order bands $I_{j}, j=1, \ldots, 4$. These conditions read $h \leq h_{c}^{(1)}$ and $f_{3}\left(x_{4}^{*}\right) \geq f_{4}\left(x_{1}^{*}\right)$, the second inequality being a condition on $H$ as well as $h$.

- The transitions depending on the scaling at the boundary of the support of $\mu_{\infty}$, i.e. on the scaling at $x_{1}^{*}$ and $x_{4}^{*}$, exist in the same way as those at $h_{c}^{(3)}$ and $h_{c}^{(4)}$ above. The transition conditions are $H=h_{c}^{(3)}$ and $H=h_{c}^{(4)}$ respectively.

- Even though the orbit structure is more intricate than in the dichotomous case figure 11 shows a situation in which a transition of the type of the one at $h_{c}^{(2)}$ above can take place. The transition occurs at $h=h_{c}^{(2)}$ provided $H$ is large enough for the bands $I_{1}$ and $I_{2}$ not to include $x_{\{23\}}^{*}$ or $x_{\{32\}}^{*}$, i.e. $f_{1}\left(x_{4}^{*}\right)<x_{\{23\}}^{*}$ or equivalently $f_{4}\left(x_{1}^{*}\right)>x_{\{32\}}^{*}$. 
In summary, the types of transitions occurring in the $D_{q}$-spectrum are the same as in the dichotomous case and it is possible to draw a diagram in the $(\beta, h, H)$ parameter space similar to the simpler diagram in figure 10 which is the $h=H$ slice of this higher dimensional diagram.

In the same fashion the analytic tools developed in this paper in principal allow to analyse the $D_{q}$-spectrum of the distribution of the effective field of the RFIM for any discrete random field distribution. Moreover, the exact form of the function $A$ is not crucial such that the analysis can be performed as soon as the following features are present:

- A hyperbolic RIFS $\left\{f_{j}\right\}, j=1, \ldots, n$ which - in dependence on a control parameter $h$ - has overlapping bands or not.

- The functions $f_{j}$ are sufficiently smooth, monotonous and have comparably large derivatives at certain periodic orbits.

- The conditions for the transitions that certain (periodic) orbits touch or do not touch the overlap can be fulfilled by tuning $h$.

The generalization to continuous random field distributions is not obvious as the techniques used above can not directly be applied to this case. In [3] a numerical survey of the nature of the distribution function $P_{\infty}$ for various random field distributions can be found. For discrete random fields a transition between a devils's staircase corresponding to $D_{0}<1$ and a smooth function corresponding to $D_{0}=1$ is found as is predicted analytically. For continuous random field distributions without gaps $P_{\infty}$ always is smooth whereas gaps in the continuous random field distribution define a scale above which (for certain parameters) $P_{\infty}$ resembles a devil's staircase and below which it is always smooth.

The generalization to more general lattices like the dichotomous symmetric RFIM on the Bethe lattice (cf. 42, 43 and references therein) is non-trivial. Transitions corresponding to those at $h_{c}^{(1)}, h_{c}^{(3)}$ and $h_{c}^{(4)}$ above are present. The exact conditions determining the critical field strengths are however not obvious and need further careful investigation. The existence and properties of transitions of the type of those at $h_{c}^{(2)}$ or $h_{c}^{(2 a)}$ needs even more careful and detailed analysis and must be deferred to further work. We stress however that all these transitions in the $D_{q}$-spectrum take place far in the contracting, paramagnetic regime of the physical phase diagram of the RFIM on the Bethe lattice.

The bounds on the $D_{q}$-spectrum obtained in section 3 are also of a quite general type and therefore applicable in a variety of contexts. As figure 2 shows, the bounds together with the explanation of all the transitions discussed give a very good qualitative as well as quantitative understanding of the $D_{q}$-spectrum of the invariant measure of the effective field in the dichotomous, symmetric 1D RFIM.

The detailed analysis of the invariant measure of the effective field should be viewed as the preparation of the study of the multifractal properties of the measure of the local magnetisation in the RFIM which essentially is a convolution of the invariant measure of the effective field with a distorted version of itself. The transitions in the $D_{q}$-spectrum of the invariant measure of the effective field have direct counterparts in the $D_{q}$-spectrum of the measure of the local magnetisation and are thus gaining a direct physical significance. We will address this subject in forthcoming work. 
Orbits and phase transitions in the multifractal spectrum

\section{Acknowledgments}

Two of us (H. P. and U. B.) acknowledge discussions with Dr. Adrian Lange in a very early stage of this work. The work was partially supported by the Graduiertenkolleg "Quantenfeldtheorie: Mathematische Struktur und Anwendungen in der Elementarteilchen- und Festkörperphysik" (DFG). Thanks are due to anonymous referees for valuable comments.

\section{Appendix A. Relations between scaling properties}

Appendix A.1. Relation between the scaling at $x_{\{\sigma\}_{n}}^{*}$ and at $f_{\{\sigma\}_{n}}^{-1}\left(x_{\{\sigma\}_{n}}^{*}\right)$

In this Appendix we show that the assumed scaling relation

$$
\lim _{\varepsilon \rightarrow 0} \frac{P_{\infty}\left(x_{\{\sigma\}_{n}}^{*}+\frac{\varepsilon}{2}\right)-P_{\infty}\left(x_{\{\sigma\}_{n}}^{*}-\frac{\varepsilon}{2}\right)}{\varepsilon^{\alpha_{\{\sigma\}_{n}}+1}}=: k
$$

implies that the limit

$$
\lim _{\varepsilon \rightarrow 0} \frac{P_{\infty}\left(f_{\{\sigma\}_{n}}^{-1}\left(x_{\{\sigma\}_{n}}^{*}+\frac{\varepsilon}{2}\right)\right)-P\left(f_{\{\sigma\}_{n}}^{-1}\left(x_{\{\sigma\}_{n}}^{*}-\frac{\varepsilon}{2}\right)\right)}{\left(\left(f_{\{\sigma\}_{n}}^{-1}\right)^{\prime}\left(x_{\{\sigma\}_{n}}^{*}\right) \varepsilon\right)^{\alpha_{\{\sigma\}_{n}}+1}}
$$

exists and is equal to $k$. In the following we drop the indices $\{\sigma\}_{n}$ and $\infty$ to improve readability and denote the expression in (A.2) as $Q(\varepsilon)$. Applying the mean value theorem to $f^{-1}$ in $Q(\varepsilon)$ and using $f^{-1}\left(x^{*}\right)=x^{*}$ yields

$$
Q(\varepsilon)=\frac{P\left(x^{*}+\left(f^{-1}\right)^{\prime}\left(x^{*}+\delta_{1}\right) \frac{\varepsilon}{2}\right)-P\left(x^{*}-\left(f^{-1}\right)^{\prime}\left(x^{*}-\delta_{2}\right) \frac{\varepsilon}{2}\right)}{\left(\left(f^{-1}\right)^{\prime}\left(x^{*}\right) \varepsilon\right)^{\alpha+1}}
$$

with some $\delta_{1}, \delta_{2} \in\left[0, \frac{\varepsilon}{2}\right]$. Now let $\left(f^{-1}\right)_{\min }^{\prime}$ be the minimum of $\left(f^{-1}\right)^{\prime}\left(x^{*}+\delta_{1}\right)$ and $\left(f^{-1}\right)^{\prime}\left(x^{*}-\delta_{2}\right)$. Because $f^{-1}$ is strictly monotonously growing we have $\left(f^{-1}\right)_{\min }^{\prime}>0$. Using the fact that $P$ is monotonously growing as well we get the lower estimate

$$
\begin{aligned}
Q(\varepsilon) & \geq \frac{P\left(x^{*}+\left(f^{-1}\right)_{\min }^{\prime} \frac{\varepsilon}{2}\right)-P\left(x^{*}-\left(f^{-1}\right)_{\min }^{\prime} \frac{\varepsilon}{2}\right)}{\left(\left(f^{-1}\right)^{\prime}\left(x^{*}\right) \varepsilon\right)^{\alpha+1}} \\
& =\frac{P\left(x^{*}+\left(f^{-1}\right)_{\min }^{\prime} \frac{\varepsilon}{2}\right)-P\left(x^{*}-\left(f^{-1}\right)_{\min }^{\prime} \frac{\varepsilon}{2}\right)}{\left(\left(f^{-1}\right)_{\min }^{\prime} \varepsilon\right)^{\alpha+1}} \cdot\left(\frac{\left(f^{-1}\right)_{\min }^{\prime} \varepsilon}{\left(f^{-1}\right)^{\prime}\left(x^{*}\right) \varepsilon}\right)^{\alpha+1} .
\end{aligned}
$$

The quotient of $\left(f^{-1}\right)_{\min }^{\prime}$ and $\left(f^{-1}\right)^{\prime}\left(x^{*}\right)$ converges to 1 and thus

$$
\lim _{\varepsilon \rightarrow 0} Q(\varepsilon) \geq \lim _{\varepsilon \rightarrow 0} \frac{P\left(x^{*}+\left(f^{-1}\right)_{\min }^{\prime} \frac{\varepsilon}{2}\right)-P\left(x^{*}-\left(f^{-1}\right)_{\min }^{\prime} \frac{\varepsilon}{2}\right)}{\left(\left(f^{-1}\right)_{\min }^{\prime} \varepsilon\right)^{\alpha+1}}=k .
$$

Using the maximum of $\left(f^{-1}\right)^{\prime}\left(x^{*}+\delta_{1}\right)$ and $\left(f^{-1}\right)^{\prime}\left(x^{*}-\delta_{2}\right)$ instead of the minimum we get in the same fashion the upper estimate

$$
\lim _{\varepsilon \rightarrow \infty} Q(\varepsilon) \leq k .
$$

Both estimates together give the conjectured result $\lim _{\varepsilon \rightarrow 0} Q(\varepsilon)=k$. 
Appendix A.2. Relation between $\alpha(x)$ and $\alpha\left(f_{\sigma}^{-1}(x)\right)$

The Frobenius-Perron equation for the invariant distribution $P_{\infty}$ induces the equality $\alpha(x)=\alpha\left(f_{\sigma}^{-1}(x)\right)$ for any $x \in \operatorname{supp} \mu$ which is not in the overlap $O$.

The proof is straightforward. Let $x \in \operatorname{supp} \mu$ be a point which is not in $O$ and $\sigma \in\{-,+\}$ an arbitrary sign. Then, dropping again the index $\infty$,

$$
\begin{aligned}
\alpha(x) & =\lim _{\varepsilon \rightarrow 0} \frac{\ln \left(P\left(x+\frac{\varepsilon}{2}\right)-P\left(x-\frac{\varepsilon}{2}\right)\right)}{\ln \varepsilon} \\
& =\lim _{\varepsilon \rightarrow 0} \frac{\ln \left(\frac{1}{2} P\left(f_{\sigma}^{-1}\left(x+\frac{\varepsilon}{2}\right)\right)-\frac{1}{2} P\left(f_{\sigma}^{-1}\left(x-\frac{\varepsilon}{2}\right)\right)\right)}{\ln \varepsilon}
\end{aligned}
$$

because of the Frobenius-Perron equation. We now use the mean value theorem for $f_{\sigma}^{-1}\left(x+\frac{\varepsilon}{2}\right)$ and $f_{\sigma}^{-1}\left(x-\frac{\varepsilon}{2}\right)$ to obtain

$$
=\lim _{\varepsilon \rightarrow 0} \frac{-\ln 2}{\ln \varepsilon}+\frac{\ln \left(P\left(f_{\sigma}^{-1}(x)+\left(f_{\sigma}^{-1}\right)^{\prime}\left(x+\delta_{1}\right) \frac{\varepsilon}{2}\right)-P\left(f_{\sigma}^{-1}(x)-\left(f_{\sigma}^{-1}\right)^{\prime}\left(x-\delta_{2}\right) \frac{\varepsilon}{2}\right)\right)}{\ln \varepsilon}
$$

with some $\delta_{1}, \delta_{2} \in\left[0, \frac{\varepsilon}{2}\right]$. Using again the notation $\left(f_{\sigma}^{-1}\right)_{\min }^{\prime}$ for the the minimum of $\left(f_{\sigma}^{-1}\right)^{\prime}\left(x+\delta_{1}\right)$ and $\left(f_{\sigma}^{-1}\right)^{\prime}\left(x-\delta_{2}\right)$ and defining $\varepsilon^{\prime}:=\left(f_{\sigma}^{-1}\right)_{\min }^{\prime} \cdot \varepsilon$ we get the inequality

$$
\alpha(x) \geq \lim _{\varepsilon^{\prime} \rightarrow 0} \frac{\ln \left(P\left(f_{\sigma}^{-1}(x)+\frac{\varepsilon^{\prime}}{2}\right)-P\left(f_{\sigma}^{-1}(x)-\frac{\varepsilon^{\prime}}{2}\right)\right)}{-\ln \left(f_{\sigma}^{-1}\right)_{\min }^{\prime}+\ln \varepsilon^{\prime}}=\alpha\left(f_{\sigma}^{-1}(x)\right) .
$$

Using the maximum $\left(f_{\sigma}^{-1}\right)_{\max }^{\prime}$ of the derivatives instead of the minimum we get by the same token $\alpha(x) \leq \alpha\left(f_{\sigma}^{-1}(x)\right)$. Therefore equality follows.

\section{Appendix B. Independence of (20) from the choice of $x_{i}^{(n)}$}

In this Appendix we show that

$$
\lim _{n \rightarrow \infty} \frac{1}{n}\left(\sum_{i=1}^{n} \ln \phi\left(x_{i}^{(n)}\right)-\sum_{i=1}^{n} \ln \phi\left(\tilde{x}_{i}^{(n)}\right)\right)=0
$$

for any strictly positive differentiable function $\phi$, any symbolic sequence $\{\sigma\}$ and any choice of $x_{i}^{(n)}, \tilde{x}_{i}^{(n)} \in I_{\{\sigma\}_{i}}, i \in\{1, \ldots, n\}, n \in \mathbb{N}$. Let $\phi,\{\sigma\}, x_{i}^{(n)}$ and $\tilde{x}_{i}^{(n)}$ be given and set $\varepsilon_{n}:=\left|I_{\{\sigma\}_{n}}\right|$. Then, using the mean value theorem,

$$
\phi\left(\tilde{x}_{i}^{(n)}\right)=\phi\left(x_{i}^{(n)}\right)+\phi^{\prime}\left(x_{i}^{(n)}+\delta_{i}^{(n)}\right)\left(\tilde{x}_{i}^{(n)}-x_{i}^{(n)}\right)
$$

with some $\delta_{i}^{(n)},\left|\delta_{i}^{(n)}\right|<\varepsilon_{i}$. Thus

$$
\ln \phi\left(\tilde{x}_{i}^{(n)}\right)-\ln \phi\left(x_{i}^{(n)}\right)=\ln \left(1+\frac{\phi^{\prime}\left(x_{i}^{(n)}+\delta_{i}^{(n)}\right)}{\phi\left(x_{i}^{(n)}\right)}\left(\tilde{x}_{i}^{(n)}-x_{i}^{(n)}\right)\right) .
$$

We denote the finite constant $\max \left\{\frac{\phi^{\prime}(x)}{\phi(x)}: x \in I\right\}$ by $Q_{\max }$. For sufficiently large $i \in \mathbb{N}$ the expression $1-Q_{\max }\left|\tilde{x}_{i}^{(n)}-x_{i}^{(n)}\right|$ is positive and we get

$$
\ln \left(1-Q_{\max }\left|\tilde{x}_{i}^{(n)}-x_{i}^{(n)}\right|\right) \leq \ln \phi\left(\tilde{x}_{i}^{(n)}\right)-\ln \phi\left(x_{i}^{(n)}\right) \leq \ln \left(1+Q_{\max }\left|\tilde{x}_{i}^{(n)}-x_{i}^{(n)}\right|\right) .
$$


With $\left|\tilde{x}_{i}^{(n)}-x_{i}^{(n)}\right| \leq \varepsilon_{i}$ this yields

$$
\ln \left(1-Q_{\max } \varepsilon_{i}\right) \leq \ln \phi\left(\tilde{x}_{i}^{(n)}\right)-\ln \phi\left(x_{i}^{(n)}\right) \leq \ln \left(1+Q_{\max } \varepsilon_{i}\right)
$$

implying that the difference $\ln \phi\left(\tilde{x}_{i}^{(n)}\right)-\ln \phi\left(x_{i}^{(n)}\right)$ converges to zero. A standard argument then shows that the average also converges to zero, i.e. that $(\bar{B} .1)$ is true. With $\phi=A^{\prime}$ this yields the alleged independence of (20) from the choice of $x_{i}^{(n)}$.

\section{References}

[1] Bruinsma R and Aeppli G 1983 Phys. Rev. Lett. 50 1494, Phys. Lett. 97 A 117

[2] Normand J M, Mehta M L and Orland H 1985 J. Phys. A 18621

[3] Andelman D 1986 Phys. Rev. B 346214

[4] Györgyi G and Rujàn P 1984 J. Phys. C 174207

[5] Behn U and Zagrebnov V A 1987 JINR, E 17-87-138 Dubna

[6] Behn U and Zagrebnov V A 1987 J. Stat. Phys. 47939

[7] Szèpfalusy P and Behn U 1987 Z. Phys. B 65337

[8] Behn U and Zagrebnov V A 1988 J. Phys. A : Math. Gen. 212151

[9] Ruján P Physica A 91549

[10] Evangelou S N 1987 J. Phys. C 20 L511

[11] Bene J and Szépfalusy P 1988 Phys. Rev. A 371703

[12] Behn U, Priezzhev V B and Zagrebnov V A 1990 Physica A 167481

[13] Behn U and Lange A 1992 From Phase Transitions to Chaos ed Györgyi G, Kondor I, Sasvári L and Tél T (Singapore: World Scientific) 217

[14] Behn U, van Hemmen J L, Kühn R, Lange A and Zagrebnov V A 1994 On Three Levels ed M Fannes et al (New York: Plenum Press) 399

[15] Patzlaff H, Behn U and Lange A 1997 FRACTAL FRONTIERS, Fractals in the Natural and Applied Sciences ed M M Novak and T G Dewey (Singapore: World Scientific) 95

[16] Bleher P M, Ruiz J and Zagrebnov V A 1996 J. Stat. Phys. 841077

[17] Luck J M and Nieuwenhuizen Th M 1989 J. Phys. A 22 2151, 1986 J. Phys. A 19

[18] Derrida B, Vannimenus J and Pomeau Y 1978 J. Phys. C 114749

[19] Tanaka T, Fujisaka H and Inoue M 1989 Phys. Rev. A 393170

[20] Tanaka T, Fujisaka H and Inoue M 1990 Prog. Theor. Phys. 84584

[21] Schmidt H 1957 Phys. Rev. 105425

[22] Halperin B I 1967 Adv. Chem. Phys. 13123

[23] Barnes C and Luck J M 1990 J. Phys. A 231717

[24] Luck J M 1992 Systèmes Desordonnés Unidimensionnéls ed C Godreche (Paris: Collection Alea-Saclay)

[25] Edalat A 1997 The Bulletin of Symbolic Logic 3401

[26] Halsey T C, Jensen M H, Procaccia I and Shraiman B I 1989 Phys. Rev. A 331141

[27] Bene J 1989 Phys. Rev. A 392090

[28] van Hemmen J L, Keller G and Kühn R 1988 Europhys. Lett. 5663

[29] Behn U, van Hemmen J L, Kühn R, Lange A, and Zagrebnov V A 1993 Physica D 68401

[30] Patzlaff H 1998 Dissertation (Universität Leipzig)

[31] Simon K, Solomyak B and Urbański M preprint http://www.math.washington.edu/ solomyak/ PREPRINTS/ssu1.ps

[32] Ledrappier F and Porzio A 1994 J. Stat. Phys. 76 1307, 1996 J. Stat. Phys. 82 367, J. Stat. Phys. 82397

[33] Falconer K J 1999 Nonlinearity 12877

[34] Hutchinson J E 1981 Indiana Univ. Math. J. 30713

[35] Elton J H 1987 Ergod. Th. E Dynam. Sys. 7481

[36] Young L S 1982 Ergod. Th. E Dynam. Sys. 2109

[37] Falconer K J 1990 Fractal Geometry (Chichester: J. Wiley \& Sons)

[38] Edalat A 1995 Theoretical Computer Science 151163

[39] Radons G 1995 Phys. Rev. Lett. 752518

[40] Radons G and Stoop R 1996 J. Stat. Phys. 821063

[41] Stoop R and Steeb F-H 1997 Phys. Rev. E 556589

[42] Bruinsma R 1984 Phys. Rev. B 30289

[43] Bleher P M, Ruiz J and Zagrebnov V A 1998 J. Stat. Phys. 9333 